DIAS-STP-09-06

pi-partphys -133

HWM-09-4

EMPG-09-7

\title{
Dimensional Reduction and Vacuum Structure of Quiver Gauge Theory
}

\author{
Brian P. Dolan ${ }^{1,2}$ and Richard J. Szabo ${ }^{3,4}$ \\ ${ }^{1}$ Department of Mathematical Physics, National University of Ireland \\ Maynooth, Co. Kildare, Ireland \\ ${ }^{2}$ Perimeter Institute for Theoretical Physics \\ 31 Caroline St. N, Waterloo, Ontario N2L 2Y5, Canada \\ ${ }^{3}$ Department of Mathematics, Heriot-Watt University \\ Colin Maclaurin Building, Riccarton, Edinburgh EH14 4AS, U.K. \\ ${ }^{4}$ Maxwell Institute for Mathematical Sciences \\ Edinburgh, U.K.
}

Email: bdolan@thphys.nuim.ie, R.J.Szabo@ma.hw.ac.uk

\begin{abstract}
We describe the structure of the vacuum states of quiver gauge theories obtained via dimensional reduction over homogeneous spaces, in the explicit example of SU(3)-equivariant dimensional reduction of Yang-Mills-Dirac theory on manifolds of the form $M \times \mathbb{C} P^{2}$. We pay particular attention to the role of topology of background gauge fields on the internal coset spaces, in this case $\mathrm{U}(1)$ magnetic monopoles and $\mathrm{SU}(2)$ instantons on $\mathbb{C} P^{2}$. The reduction of Yang-Mills theory induces a quiver gauge theory involving coupled Yang-Mills-Higgs systems on $M$ with a Higgs potential leading to dynamical symmetry breaking. The criterion for a ground state of the Higgs potential can be written as the vanishing of a non-abelian Yang-Mills flux on the quiver diagram, regarded as a lattice with group elements attached to the links. The reduction of $\mathrm{SU}(3)$-symmetric fermions yields Dirac fermions on $M$ transforming under the low-energy gauge group with Yukawa couplings. The fermionic zero modes on $\mathbb{C} P^{2}$ yield exactly massless chiral fermions on $M$, though there is a unique choice of $\operatorname{spin}^{c}$ structure on $\mathbb{C} P^{2}$ for which some of the zero modes can acquire masses through Yukawa interactions. We work out the spontaneous symmetry breaking patterns and determine the complete physical particle spectrum in a number of explicit examples, some of which possess quantum number assignments qualitatively analogous to the manner in which vector bosons, quarks and leptons acquire masses in the standard model.
\end{abstract}




\section{Introduction}

The Kaluza-Klein programme, i.e. the idea that the Higgs and Yukawa sectors of the standard model of particle physics could have their origins in a simpler but higher-dimensional theory, remains as attractive today as when it was when first proposed [1]. In the original idea of Kaluza and Klein, and its non-abelian generalisation with a homogeneous internal space $G / H$ for $H$ a closed subgroup of a compact Lie group $G$, the higher-dimensional theory was pure gravity but in later schemes Einstein-Yang-Mills theories in higher dimensions were introduced [2]. This has the potential to provide a unification of the gauge and Higgs sectors in higher dimensions, while the coupling of fermions to the higher-dimensional gauge theory naturally induces Yukawa couplings after dimensional reduction. For certain coset spaces, particularly the complex projective plane, the inclusion of topologically non-trivial internal fluxes can induce the chiral fermionic spectrum of quarks and leptons of the standard model [3].

The pioneering scheme realizing these constructions is called "coset space dimensional reduction" [4, 5], though a generic problem with such reductions has been that they are unable to generate chiral gauge theories, without some additional modifications [5, 6. In coset space dimensional reduction, constraints are imposed on the higher-dimensional fields ensuring that they are invariant under the $G$-action up to gauge transformations. This amounts to studying embeddings of the isometry group $G$ of the coset space, or of its holonomy subgroup $H$, in the gauge group of the higher-dimensional theory and the solutions of the constraints are then formally identified with the lowest modes of the harmonic towers of fields.

On the other hand, the "equivariant dimensional reduction" of gauge theories naturally incorporates the topology of background fields on $G / H$ which are gauged with respect to the holonomy group $H$. Although similar in spirit to the coset space dimensional reduction scheme, equivariant dimensional reduction systematically constructs the unique field configurations on the higherdimensional space which are equivariant with respect to the internal isometry group $G$, and reduces Yang-Mills theory to a quiver gauge theory based on a quiver (with relations) which is determined entirely by the representation theory of the Lie groups $G$ and $H$. As in coset space dimensional reduction, there is no a priori relation between the gauge group $\mathcal{G}$ of the higher-dimensional field theory and the groups $G$ or $H$, and the resulting gauge group of the dimensionally reduced field theory is a subgroup of $\mathcal{G}$. This differs from the usual Kaluza-Klein reductions where the isometry group (or the holonomy group) is identified with the gauge group. The general formalism is described in [7, 8. It has been applied in a variety of contexts in [9, 10, when the internal coset space is the projective line $\mathbb{C} P^{1}$. Dimensional reduction over the fuzzy sphere $\mathbb{C} P_{F}^{1}$ is also considered in [10, 11]. In this paper we extend the analysis of the vacuum states of such quiver gauge theories performed in [10] to an example with non-abelian holonomy, the projective plane $\mathbb{C} P^{2}$. The corresponding quiver gauge theories have been discussed in [12]. This example is rich enough to capture some general features of the vacua of the quiver gauge theories which are induced by reduction over generic coset spaces $G / H$.

When the internal space is the projective plane $\mathbb{C} P^{2}$, the equivariant dimensional reduction of gauge fields naturally comes with $\mathrm{U}(1)$ monopoles and $\mathrm{SU}(2)$ instantons, in contrast to $\mathbb{C} P^{1}$ where only monopoles are present, and this introduces essential differences from the $\mathbb{C} P^{1}$ case. As in the Kaluza-Klein approach, the mass scale of the dimensionally reduced field theory is set by the size of the internal space. We obtain a Higgs sector of the lower-dimensional gauge theory with a Higgs potential that leads to dynamical symmetry breaking, as a direct consequence of the non-trivial internal fluxes, and we work out the complete physical particle content and masses for a number of explicit symmetry breaking hierarchies. As in the case of reduction over $\mathbb{C} P^{1}$, a Yukawa sector of the reduced fermionic field theory is naturally induced. The harmonic expansion over $\mathbb{C} P^{2}$ induces an infinite tower of massive fermions in the reduced field theory, but the topologically non-trivial 
gauge fields on the internal $\mathbb{C} P^{2}$ necessarily also induce exactly massless chiral modes in the reduced field theory. As in the $\mathbb{C} P^{1}$ case, some of the massless spinor fields which arise as a consequence of the index theorem on the internal space acquire masses through their Yukawa couplings, but in general not all of them.

There is a number of other differences between the equivariant dimensional reduction over $\mathbb{C} P^{1}$ and that over $\mathbb{C} P^{2}$ which is studied here. The fact that the rank of the holonomy group is now greater than one means that the quiver diagram is no longer a one-dimensional chain but is a higher-dimensional lattice, of dimension two in the case of $\mathbb{C} P^{2}$. We show that the condition for a vacuum state of the Higgs sector of the reduced field theory can be phrased in terms of a nonabelian gauge theory on the quiver lattice. A group element associated with the Higgs field can be placed on each link of the quiver diagram, and minimising the Higgs potential requires that the resulting gauge field flux on the quiver lattice is zero. The Higgs vacuum requires that the lattice gauge field is gauge equivalent to the trivial gauge potential.

Another difference is associated with spinors on $\mathbb{C} P^{2}$ and the treatment of the fermionic field theory. It is well-known that $\mathbb{C} P^{2}$ does not admit a spin structure, as there is a global obstruction to putting spinors on $\mathbb{C} P^{2}$ associated with the fact that its second Stiefel-Whitney class is non-trivial [13]. However, since the equivariant dimensional reduction scheme necessarily induces topologically non-trivial monopole and instanton fields on the internal space, the reduction itself provides a solution to the problem of absence of spin structure on $\mathbb{C} P^{2}$ by simply coupling spinor fields to non-trivial gauge backgrounds and using $\operatorname{spin}^{c}$ structures for line bundles or nonabelian $\operatorname{spin}^{c}$ structures for higher rank bundles. Gauge fields on $\mathbb{C} P^{2}$ and their coupling to spinors were studied in [14, 15]. We will find that there is a unique $\operatorname{spin}^{c}$ structure accommodating the background gauge fields on $\mathbb{C} P^{2}$ which generically lead to Yukawa interactions after dimensional reduction, in contrast to the $\mathbb{C} P^{1}$ reductions, whereas other choices of twisting can produce more realistic generations of fermions. Altogether, we will explicitly display models in which the quantum number assignments for the fermions are qualitatively similar to those of quarks and leptons in the standard model.

This paper is organised as follows. In $₫ 2$ we describe the kinematics of equivariant dimensional reduction over $\mathbb{C} P^{2}$, particularly how the gauge and Higgs fields in the reduced field theory depend on representation theory and the various irreducible $\mathrm{SU}(2) \times \mathrm{U}(1)$ representations that can arise from a given $\mathrm{SU}(3)$ representation, as well as the harmonic expansion of zero mode spinors. In $\$ 3$ we derive the dimensionally reduced action, showing how the Higgs potential depends on the group representation content and how Yukawa couplings are induced in the Dirac action. Some examples are studied in detail in the ensuing two sections, one class of examples based on the fundamental representation of $\mathrm{SU}(3)$ in $\$ 4$ and one class based on the adjoint representation in $\$ 5$, Our conclusions are summarised in 96 . Some technical details are relegated to three appendices at the end of the paper. In $₫$ we calculate Chern numbers for the various equivariant vector bundles over $\mathbb{C} P^{2}$ required in our analysis. Some useful identities for equivariant one-forms on $\mathbb{C} P^{2}$ are given in $\S \mathrm{B}$. Finally, the index of the Dirac operator on $\mathbb{C} P^{2}$, coupled to various topologically non-trivial gauge field backgrounds, is computed in

\section{Equivariant dimensional reduction over the projective plane}

In this section we will describe the $\mathrm{SU}(3)$-equivariant dimensional reduction of gauge and fermion fields over an internal complex projective plane $\mathbb{C} P^{2}$. For some further details, see [12]. Throughout this section all local coordinates and fields are taken to be dimensionless. 


\subsection{Homogeneous vector bundles on $\mathbb{C} P^{2}$}

We are interested in the geometry of the symmetric coset space $\mathbb{C} P^{2} \cong G / H$, where

$$
H=\mathrm{S}(\mathrm{U}(2) \times \mathrm{U}(1)) \cong \mathrm{SU}(2) \times \mathrm{U}(1)
$$

is the holonomy subgroup of the isometry group $G=\mathrm{SU}(3)$ of $\mathbb{C} P^{2}$. Given a finite-dimensional representation $\underline{V}$ of $H$, the corresponding induced, homogeneous hermitean vector bundle over $\mathbb{C} P^{2}$ is given by the fibred product

$$
\mathcal{V}=G \times_{H} \underline{V} .
$$

Every $G$-equivariant bundle of finite rank over $\mathbb{C} P^{2}$, with respect to the standard left transitive action of $G$ on the homogeneous space, is of the form (2.2). If $\underline{V}$ is irreducible, then $H$ is the structure group of the associated principal bundle. We restrict to those representations $\underline{V}$ which descend from some irreducible representation of $\mathrm{SU}(3)$ by restriction to $H$.

The Dynkin diagram for $\mathrm{SU}(3)$ consists of a pair of roots $\alpha_{1}, \alpha_{2}$. The complete set $\Delta$ of non-null roots is $\pm \alpha_{1}, \pm \alpha_{2}, \pm\left(\alpha_{1}+\alpha_{2}\right)$, with the inner products $\left(\alpha_{1}, \alpha_{1}\right)=\left(\alpha_{2}, \alpha_{2}\right)=1$ and $\left(\alpha_{1}, \alpha_{2}\right)=-\frac{1}{2}$ so that $\left(\alpha_{1}+\alpha_{2}, \alpha_{1}+\alpha_{2}\right)=1$. For the system $\Delta^{+}$of positive roots we take $\alpha_{1}=(1,0), \alpha_{2}=\frac{1}{2}(-1, \sqrt{3})$ and $\alpha_{1}+\alpha_{2}=\frac{1}{2}(1, \sqrt{3})$. The generators of $\mathrm{SU}(3)$ for the CartanWeyl basis are given by the Chevalley generators $E_{\alpha_{1}}, E_{\alpha_{2}}$ and $E_{\alpha_{1}+\alpha_{2}}:=\left[E_{\alpha_{1}}, E_{\alpha_{2}}\right]$, together with the generators $H_{\alpha_{1}}$ and $H_{\alpha_{2}}$ of the Cartan subalgebra $\mathrm{u}(1) \oplus \mathrm{u}(1)$. The non-vanishing commutation relations are

$$
\begin{array}{rll}
{\left[H_{\alpha_{1}}, E_{ \pm \alpha_{1}}\right]= \pm 2 E_{ \pm \alpha_{1}}} & \text { and } & {\left[H_{\alpha_{2}}, E_{ \pm \alpha_{1}}\right]=0} \\
{\left[H_{\alpha_{1}}, E_{ \pm \alpha_{2}}\right]=\mp E_{ \pm \alpha_{2}}} & \text { and } & {\left[H_{\alpha_{2}}, E_{ \pm \alpha_{2}}\right]= \pm 3 E_{ \pm \alpha_{2}}} \\
{\left[H_{\alpha_{1}}, E_{ \pm\left(\alpha_{1}+\alpha_{2}\right)}\right]= \pm E_{ \pm\left(\alpha_{1}+\alpha_{2}\right)}} & \text { and } & {\left[H_{\alpha_{2}}, E_{ \pm\left(\alpha_{1}+\alpha_{2}\right)}\right]= \pm 3 E_{ \pm\left(\alpha_{1}+\alpha_{2}\right)}} \\
{\left[E_{\alpha_{1}}, E_{-\alpha_{1}}\right]=H_{\alpha_{1}}} & \text { and } & {\left[E_{\alpha_{2}}, E_{-\alpha_{2}}\right]=\frac{1}{2}\left(H_{\alpha_{2}}-H_{\alpha_{1}}\right)} \\
{\left[E_{\alpha_{1}+\alpha_{2}}, E_{-\alpha_{1}-\alpha_{2}}\right]=\frac{1}{2}\left(H_{\alpha_{1}}+H_{\alpha_{2}}\right)} & \text { and } & {\left[E_{ \pm \alpha_{1}}, E_{ \pm \alpha_{2}}\right]=E_{ \pm\left(\alpha_{1}+\alpha_{2}\right)}} \\
{\left[E_{ \pm \alpha_{1}}, E_{\mp\left(\alpha_{1}+\alpha_{2}\right)}\right]=\mp E_{\mp \alpha_{2}}} & \text { and } & {\left[E_{ \pm \alpha_{2}}, E_{\mp\left(\alpha_{1}+\alpha_{2}\right)}\right]= \pm E_{\mp \alpha_{1}} .}
\end{array}
$$

The fundamental weights are $\mu_{\alpha_{1}}=\frac{1}{2}\left(1, \frac{1}{\sqrt{3}}\right)$ and $\mu_{\alpha_{2}}=\left(0, \frac{1}{\sqrt{3}}\right)$. For each pair of non-negative integers $(k, l)$ there is an irreducible representation $\underline{C}^{k, l}$ of $\mathrm{SU}(3)$ of dimension

$$
d^{k, l}:=\operatorname{dim}\left(\underline{C}^{k, l}\right)=\frac{1}{2}(k+1)(l+1)(k+l+2)
$$

and highest weight $\mu=k \mu_{\alpha_{1}}+l \mu_{\alpha_{2}}$. We label the weight vectors of $\mathrm{U}(2) \cong \mathrm{SU}(2) \times \mathrm{U}(1)$ in $\mathrm{SU}(3)$ by $(n, m)$ with respect to the basis $\left(H_{\alpha_{1}}, H_{\alpha_{2}}\right)$. The eigenvalue of $H_{\alpha_{1}}$ is $n=2 I$ and labels twice the isospin $I$, so that $(n+1)$ is the dimension of the irreducible $\mathrm{SU}(2)$ representation. The eigenvalue $m=3 Y$ of $H_{\alpha_{2}}$ is three times the hypercharge $Y$, and later on we shall identify $m$ with twice the magnetic charge. The restriction of the $\mathrm{SU}(3)$ operators $E_{ \pm \alpha_{1}}$ to $\mathrm{SU}(2)$ shifts vertices along the horizontal directions of the weight diagrams, while the generators $E_{\alpha_{2}}$ and $E_{\alpha_{1}+\alpha_{2}}$ act on the weights as

$$
(n, m) \longmapsto(n \pm 1, m+3)
$$

depending on which particular weight vectors $(n, m)$ the raising operators $E_{\alpha_{1}+\alpha_{2}}$ and $E_{\alpha_{2}}$ act on.

For a fixed pair of non-negative integers $(k, l)$, the decomposition of the irreducible $\mathrm{SU}(3)$ module $\underline{C}^{k, l}$ as a representation of $\mathrm{SU}(2) \times \mathrm{U}(1)$ can be obtained by collapsing the "horizontal" $\mathrm{SU}(2)$ representations to single nodes in the weight diagram for $\underline{C}^{k, l}$. The corresponding collection 
of weights $(n, m)$, which we denote by $\mathrm{W}_{k, l}$, is conveniently parameterized by a pair of independent $\mathrm{SU}(2)$ spins $j_{ \pm}=j_{ \pm}(n, m)$, with $2 j_{+}=0,1, \ldots, k$ and $2 j_{-}=0,1, \ldots, l$, that are defined in terms of Young tableaux as follows. Represent the irreducible $H$-module $(n, m)$ with $(n, m)=(1,1)$ by $\square$ and that with $(n, m)=(0,-2)$ by $\square$. Then the $\mathrm{SU}(3) \rightarrow \mathrm{SU}(2) \times \mathrm{U}(1)$ decomposition of the fundamental representation

$$
\left.\underline{C}^{1,0}\right|_{H}=\underline{(1,1)} \oplus \underline{(0,-2)}
$$

is depicted by

$$
\square \quad \longrightarrow \quad x_{1} \oplus 0_{-2}
$$

In terms of SU(3) Young tableaux, the irreducible representation $\underline{C}^{k, l}$ corresponds to the diagram

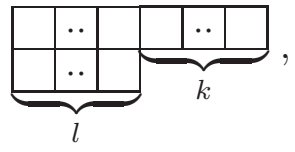

and this contains all $\mathrm{SU}(2) \times \mathrm{U}(1)$ representations

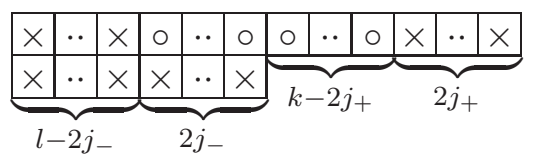

of dimension $2 j_{+}+2 j_{-}+1$ and charge $2(l-k)+6\left(j_{+}-j_{-}\right)$, with multiplicity one. This gives

$$
n=2\left(j_{+}+j_{-}\right) \quad \text { and } \quad m=6\left(j_{+}-j_{-}\right)-2(k-l) .
$$

The integers $(n, m)$ have the same even/odd parity. This is because the weights come from embedding $\mathrm{SU}(2) \times \mathrm{U}(1)$ in $\mathrm{SU}(3)$, and as such they only give faithful representations of $\mathrm{U}(2)$.

The bundle (2.2) with $\underline{V}=\left.\underline{C}^{k, l}\right|_{H}$ corresponds to a representation of a certain finite quiver with relations [12] in the category of homogeneous vector bundles over $\mathbb{C} P^{2}$. The elements $(n, m)$ of the set $\mathrm{W}_{k, l}$ can be associated with vertices of a directed graph

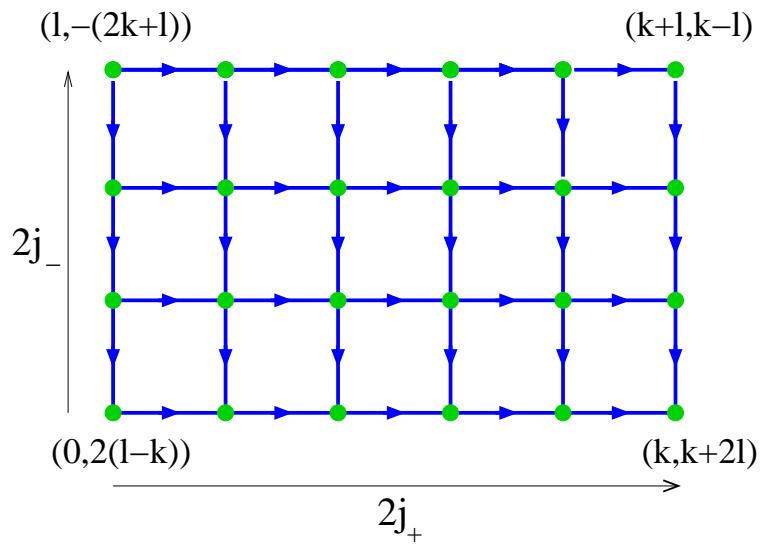

where only the four boundary corners are labelled with their values of $(n, m)$ to avoid cluttering the diagram. The weight morphisms (2.5) take the simple forms

$$
\begin{aligned}
& j_{+}(n+1, m+3)=j_{+}(n, m)+\frac{1}{2} \\
& j_{-}(n-1, m+3)=j_{-}(n, m)-\frac{1}{2}
\end{aligned} \quad \text { and } \quad \begin{aligned}
& j_{+}(n-1, m+3)=j_{+}(n, m), \\
& j_{-}(n+1, m+3)=j_{-}(n, m),
\end{aligned}
$$

corresponding to the horizontal and vertical arrows in (2.11). We will refer to this graph as the "quiver lattice", since the vacua of the quiver gauge theories we consider later on have an elegant interpretation in terms of lattice gauge theory defined on the directed graph (2.11). 


\section{2 $\mathrm{SU}(3)$-equivariant bundles}

We are interested in the structure of $G$-equivariant gauge fields on manifolds of the form

$$
\mathcal{M}:=M \times \mathbb{C} P^{2}=G \times{ }_{H} M,
$$

where $M$ is a manifold of (real) dimension $d$ and $G=\mathrm{SU}(3)$ acts trivially on $M$. We will reduce gauge theory on (2.13) by compensating the isometries of $\mathbb{C} P^{2}$ with gauge transformations, such that the Lie derivative with respect to a Killing vector field is given by an infinitesimal gauge transformation on $\mathcal{M}$. This twisted reduction is accomplished by uniquely extending the homogeneous vector bundles (2.2) by $H$-equivariant bundles $E \rightarrow M$.

Let $\mathcal{E}^{k, l} \rightarrow \mathcal{M}$ be a rank $p$ hermitean vector bundle over the space (2.13), associated to an irreducible representation $\underline{C}^{k, l}$ of $\mathrm{SU}(3)$, with structure group $\mathrm{U}(p)$. There is a one-to-one correspondence between $G$-equivariant hermitean vector bundles over $\mathcal{M}$ and $H$-equivariant hermitean vector bundles over $M$, with $H$ acting trivially on $M$ [7]. Given an $H$-equivariant bundle $E^{k, l} \rightarrow M$ of rank $p$ associated to the representation $\left.\underline{C}^{k, l}\right|_{H}$ of $H$, the corresponding $G$-equivariant bundle over $\mathcal{M}$ is defined by induction as

$$
\mathcal{E}^{k, l}=G \times{ }_{H} E^{k, l} .
$$

The action of the holonomy group $H$ on $E^{k, l}$ is defined by the isotopical decomposition

$$
E^{k, l}=\bigoplus_{(n, m) \in \mathrm{W}_{k, l}} E_{n, m} \otimes \underline{(n, m)} \quad \text { with } \quad E_{n, m}=\operatorname{Hom}_{H}\left(\underline{(n, m)}, E^{k, l}\right)
$$

where $\underline{(n, m)}$ are the irreducible $H$-modules occurring in the decomposition of $\left.\underline{C}^{k, l}\right|_{H}$. The vector bundles $\overline{E_{n, m}} \rightarrow M$ have rank $p_{n, m}$ and trivial $H$-actions. The rank $p$ of $E^{k, l}$ is given by

$$
p=\sum_{(n, m) \in \mathrm{W}_{k, l}}(n+1) p_{n, m}
$$

The action of the $\mathrm{SU}(3)$ operators $E_{ \pm \alpha_{2}}$ and $E_{ \pm\left(\alpha_{1}+\alpha_{2}\right)}$ is implemented by means of bifundamental Higgs fields $\phi_{n, m}^{ \pm} \in \operatorname{Hom}\left(E_{n, m}, E_{n \pm 1, m+3}\right)$. These bundle morphisms realize the $G$-action of the coset generators which twists the naive dimensional reduction by "off-diagonal" terms. This construction explicitly breaks the gauge group of the bundle $E^{k, l}$ as

$$
\mathrm{U}(p) \longrightarrow \prod_{(n, m) \in \mathrm{W}_{k, l}} \mathrm{U}\left(p_{n, m}\right)
$$

With

$$
\mathcal{H}_{n, m}=G \times_{H} \underline{(n, m)}
$$

the homogeneous bundle (2.2) induced by the irreducible $H$-module $(n, m)$, the structure group of the principal bundle associated to

$$
\mathcal{E}^{k, l}=\bigoplus_{(n, m) \in \mathrm{W}_{k, l}} E_{n, m} \otimes \mathcal{H}_{n, m}
$$

is then $H \times \prod_{(n, m) \in \mathrm{W}_{k, l}} \mathrm{U}\left(p_{n, m}\right)$. 


\subsection{Canonical connections on $\mathbb{C} P^{2}$}

Let us describe the unique $G$-equivariant connection on the vector bundles associated with the principal $H$-bundle

$$
\mathrm{SU}(3) \stackrel{\mathrm{S}(\mathrm{U}(2) \times \mathrm{U}(1))}{\longrightarrow} \mathbb{C} P^{2} .
$$

The projective plane can be covered by three patches, and on one of these patches we choose complex coordinates

$$
Y:=\left(\begin{array}{l}
y^{1} \\
y^{2}
\end{array}\right) \quad \text { and } \quad Y^{\dagger}=\left(\bar{y}^{1} \bar{y}^{2}\right)
$$

with $Y^{\dagger} Y=\bar{y}^{i} y^{i}$ and $i=1,2$. Introduce the column one-form

$$
\bar{\beta}:=\left(\begin{array}{l}
\bar{\beta}^{1} \\
\bar{\beta}^{2}
\end{array}\right) \quad \text { with } \quad \bar{\beta}^{i}=\frac{1}{\gamma} \mathrm{d} \bar{y}^{i}-\frac{\bar{y}^{i}}{\gamma^{2}(\gamma+1)} y^{j} \mathrm{~d} \bar{y}^{j},
$$

where

$$
\gamma:=\sqrt{1+\bar{y}^{i} y^{i}}
$$

The (1,0)-forms $\beta^{i}$ and the $(0,1)$-forms $\bar{\beta}^{i}$ constitute a $G$-equivariant basis for the complex vector spaces of forms of type $(1,0)$ and $(0,1)$ on $\mathbb{C} P^{2}$, respectively, and give the horizontal components of a flat connection $A_{0}$ tangent to the base of the bundle (2.20) [12].

Consider the $G$-equivariant field given by

$$
a=-\frac{1}{2 \gamma^{2}}\left(y^{i} \mathrm{~d} \bar{y}^{i}-\bar{y}^{i} \mathrm{~d} y^{i}\right)
$$

The one-form (2.24) is the $\mathrm{u}(1)$-valued monopole potential on $\mathbb{C} P^{2}$ which can be described as the canonical abelian connection on the Hopf bundle

$$
S^{5}=\mathrm{U}(3) / \mathrm{U}(2) \stackrel{\mathrm{U}(1)}{\longrightarrow} \mathbb{C} P^{2} .
$$

The complex line bundle $\mathcal{L} \rightarrow \mathbb{C} P^{2}$ associated with the principal U(1)-bundle (2.25) is the monopole bundle over $\mathbb{C} P^{2}$ which we take to be endowed with the same $\mathrm{u}(1)$-connection $a$. It is a representative of the isomorphism class in $\mathrm{H}^{1}\left(\mathbb{C} P^{2} ; \mathrm{U}(1)\right) \cong \mathbb{Z}$ corresponding to the abelian field strength

$$
f_{\mathrm{u}(1)}:=\mathrm{d} a=\bar{\beta}^{1} \wedge \beta^{1}+\bar{\beta}^{2} \wedge \beta^{2} .
$$

Higher degree monopole bundles $\mathcal{L}_{m / 2}:=\left(\mathcal{L}^{\otimes m}\right)^{1 / 2}$ are endowed with the connection $\frac{m}{2} a$. These bundles are associated to higher weight irreducible representations $(m)$ of the fibres of (2.25) but only exist globally when $m$ is even, as only then is the first Chern number $\frac{m}{2}$ an integer. Nevertheless, odd values of $m$ are necessary for construction of the $\mathrm{U}(2)$ bundle $\mathcal{Q}$ with curvature $F_{\mathrm{u}(2)}$ below and, as we shall see, for considering invariant spinors. The justification for calling $\frac{m}{2}$ the "monopole charge" is explained in $\$$, The monopole field strength of charge $\frac{m}{2}$ is a $(1,1)$-form proportional to the canonical Kähler two-form on $\mathbb{C} P^{2}$ defined by

$$
\omega=\mathrm{i} R^{2}\left(\beta^{1} \wedge \bar{\beta}^{1}+\beta^{2} \wedge \bar{\beta}^{2}\right),
$$

where $R$ is the radius of the linearly embedded projective line $\mathbb{C} P^{1} \subset \mathbb{C} P^{2}$ whose homology class is Poincaré dual to the cohomology class of (2.26).

Consider now the $G$-equivariant field $B \in \mathrm{u}(2)$ defined by

$$
B=\frac{1}{\gamma^{2}}\left(-\frac{1}{2} \mathrm{~d}\left(Y^{\dagger} Y\right) \mathbf{1}_{2}+\bar{Y} \mathrm{~d} \bar{Y}^{\dagger}+\Lambda \mathrm{d} \Lambda\right)
$$


where

$$
\Lambda:=\gamma \mathbf{1}_{2}-\frac{1}{\gamma+1} Y Y^{\dagger}
$$

The one-forms $B-\frac{1}{2} \operatorname{tr}(B) \mathbf{1}_{2}$ and $a$ on $\mathbb{C} P^{2}$ give the vertical components of $A_{0}$ with values in the tangent space $\mathrm{su}(2) \oplus \mathrm{u}(1)$ to the fibre of the bundle (2.20), and together with the forms (2.22) they obey the Cartan-Maurer equations

$$
\mathrm{d} \bar{\beta}+B \wedge \bar{\beta}+2 a \wedge \bar{\beta}=0 \quad \text { and } \quad \mathrm{d} \beta-B \wedge \beta-2 a \wedge \beta=0 .
$$

The $\mathrm{u}(2)$-valued curvature

$$
F_{\mathrm{u}(2)}:=\mathrm{d} B+B \wedge B=\bar{\beta} \wedge \beta^{\top}=F_{\mathrm{su}(2)}+\frac{1}{2} f_{\mathrm{u}(1)} \mathbf{1}_{2}
$$

can be expressed in terms of the abelian field strength (2.26) and the curvature

$$
F_{\mathrm{su}(2)}:=\left(\begin{array}{cc}
\frac{1}{2}\left(\bar{\beta}^{1} \wedge \beta^{1}-\bar{\beta}^{2} \wedge \beta^{2}\right) & \bar{\beta}^{1} \wedge \beta^{2} \\
\bar{\beta}^{2} \wedge \beta^{1} & -\frac{1}{2}\left(\bar{\beta}^{1} \wedge \beta^{1}-\bar{\beta}^{2} \wedge \beta^{2}\right)
\end{array}\right)=\mathrm{d} B_{(1)}+B_{(1)} \wedge B_{(1)}
$$

of the gauge potential $B_{(1)}=B-\frac{1}{2} a \mathbf{1}_{2} \in \mathrm{su}(2)$. The one-form $B_{(1)}$ is the su(2)-valued oneinstanton field on $\mathbb{C} P^{2}$ considered as the canonical connection on the $\mathrm{SU}(2)$-bundle

$$
\mathcal{I}=\mathcal{I}_{1}=S^{5} \times_{\rho} \mathrm{SU}(2)
$$

associated to the Hopf bundle (2.25) by the diagonal embedding $\rho: \mathrm{U}(1) \rightarrow \mathrm{SU}(2)$. Its curvature $F_{\mathrm{su}(2)}$ is also a $(1,1)$-form on $\mathbb{C} P^{2}$. Higher rank instanton bundles $\mathcal{I}_{n}$ are endowed with $G$-equivariant one-instanton connections $B_{(n)} \in \mathrm{su}(n+1)$ and fibre spaces in $(n+1)$-dimensional irreducible representations of the $\mathrm{SU}(2)$ fibres of the bundle (2.33). As explained in $9 \mathrm{~A}$, the bundle $\mathcal{I}_{n}$ is only globally defined for even values of $n$. For a given representation $(n, m)$ of the holonomy group $H=\mathrm{S}(\mathrm{U}(2) \times \mathrm{U}(1))$, the corresponding homogeneous vector bundle (2.2) is given by (2.18) and can be identified with $\mathcal{I}_{n} \otimes \mathcal{L}_{m / 2}$.

\subsection{Invariant gauge fields}

To determine the generic form of a $G$-equivariant connection one-form $\mathcal{A}$ on the vector bundle $\mathcal{E}^{k, l} \rightarrow \mathcal{M}$, let us assume for simplicity that $M$ (and hence $\mathcal{M}$ ) is a complex manifold. We decompose the space $\Omega^{0,1}\left(\operatorname{End}\left(\mathcal{E}^{k, l}\right)\right)^{G}$ using the Whitney sum (2.19). By Schur's lemma, corresponding to each weight $(n, m) \in \mathrm{W}_{k, l}$ there is a "diagonal" subspace

$$
\left(\Omega^{0,1}\left(\operatorname{End}\left(E_{n, m}\right)\right) \otimes \mathbf{1}_{n+1}\right) \oplus\left(\mathbf{1}_{p_{n, m}} \otimes \Omega^{0,1}\left(\operatorname{End}\left(\mathcal{H}_{n, m}\right)\right)^{G}\right),
$$

in which we can choose a connection $A^{n, m}$ on the bundle $E_{n, m} \rightarrow M$ twisted by a $G$-equivariant connection on the homogeneous vector bundle $\mathcal{H}_{n, m} \rightarrow \mathbb{C} P^{2}$ constructed from the gauge potentials $a$ and $B_{(1)}$ of 92.3 . To each weight morphism (2.5) there is an "off-diagonal" subspace

$$
\Omega^{0}\left(\operatorname{Hom}\left(E_{n, m}, E_{n \pm 1, m+3}\right)\right) \otimes \Omega^{0,1}\left(\operatorname{Hom}\left(\mathcal{H}_{n, m}, \mathcal{H}_{n \pm 1, m+3}\right)\right)^{G}
$$

in which we twist the Higgs fields $\phi_{n, m}^{ \pm}$by suitable invariant $(n \pm 1+1) \times(n+1)$ matrix-valued $(0,1)$-forms built from the basis $(0,1)$-forms $\bar{\beta}^{i}$ spanning $\Omega^{0,1}\left(\mathbb{C} P^{2}\right)^{G}$ that were constructed in 92.3 . Thus the condition of $G$-equivariance uniquely dictates the form of the gauge connection $\mathcal{A}$ in $(n+1) p_{n, m} \times(n \pm 1+1) p_{n \pm 1, m+3}$ blocks.

To appropriately assemble the invariant $(0,1)$-forms into rectangular block matrices, we will use the Biedenharn basis for the irreducible representations $\underline{C}^{k, l}$ of SU(3). The complete set of $d^{k, l}$ 
orthonormal vectors in this basis set are denoted $\left|{ }_{q}^{n}, m\right\rangle$, and are labelled by the isospin quantum numbers $n=2 I, q=2 I_{z}$ and the hypercharge $m=3 Y$. These states define the spin $\frac{n}{2}$ representation of the isospin subgroup $\mathrm{SU}(2) \subset \mathrm{SU}(3)$ and are hypercharge eigenstates with the matrix elements

$$
\begin{aligned}
& \left.\left.\left.H_{\alpha_{1}}\right|_{q} ^{n}, m\right\rangle=\left.q\right|_{q} ^{n}, m\right\rangle, \\
& E_{ \pm \alpha_{1}}|\stackrel{n}{q}, m\rangle=\frac{1}{2} \sqrt{(n \mp q)(n \pm q+2)}\left|\begin{array}{c}
n \\
q \pm 2
\end{array}, m\right\rangle \\
& \left.\left.H_{\alpha_{2}}\right|_{q} ^{n}, m\right\rangle=m|\stackrel{n}{q}, m\rangle, \\
& \left.\left.\left.\left.E_{\alpha_{2}}\right|_{q} ^{n}, m\right\rangle=\left.E_{\alpha_{2}}^{+}\right|_{q} ^{n}, m\right\rangle+\left.E_{\alpha_{2}}^{-}\right|_{q} ^{n}, m\right\rangle \\
& \left.\left.:=\left.\sqrt{\frac{n-q+2}{2(n+1)}} \Lambda_{k, l}^{+}(n, m)\right|_{q-1} ^{n+1}, m+3\right\rangle+\left.\sqrt{\frac{n+q}{2(n+1)}} \Lambda_{k, l}^{-}(n, m)\right|_{q-1} ^{n-1}, m+3\right\rangle, \\
& \left.\left.\left.\left.E_{\alpha_{1}+\alpha_{2}}\right|_{q} ^{n}, m\right\rangle=\left.E_{\alpha_{1}+\alpha_{2}}^{+}\right|_{q} ^{n}, m\right\rangle+\left.E_{\alpha_{1}+\alpha_{2}}^{-}\right|_{q} ^{n}, m\right\rangle \\
& \left.\left.:=\left.\sqrt{\frac{n+q+2}{2(n+1)}} \Lambda_{k, l}^{+}(n, m)\right|_{q+1} ^{n+1}, m+3\right\rangle+\left.\sqrt{\frac{n-q}{2(n+1)}} \Lambda_{k, l}^{-}(n, m)\right|_{q+1} ^{n-1}, m+3\right\rangle,
\end{aligned}
$$

where

$$
\begin{aligned}
& \Lambda_{k, l}^{+}(n, m)=\frac{1}{\sqrt{n+2}} \sqrt{\left(\frac{k+2 l}{3}+\frac{n}{2}+\frac{m}{6}+2\right)\left(\frac{k-l}{3}+\frac{n}{2}+\frac{m}{6}+1\right)\left(\frac{2 k+l}{3}-\frac{n}{2}-\frac{m}{6}\right)} \\
& \Lambda_{k, l}^{-}(n, m)=\frac{1}{\sqrt{n}} \sqrt{\left(\frac{k+2 l}{3}-\frac{n}{2}+\frac{m}{6}+1\right)\left(\frac{l-k}{3}+\frac{n}{2}-\frac{m}{6}\right)\left(\frac{2 k+l}{3}+\frac{n}{2}-\frac{m}{6}+1\right)} .
\end{aligned}
$$

The latter constants are defined for $n>0$ and we set $\Lambda_{k, l}^{-}(0, m):=0$. The analogous relations for $E_{-\alpha_{2}}$ and $E_{-\alpha_{1}-\alpha_{2}}$ can be derived by hermitean conjugation of (2.39) and (2.40), respectively.

For a fixed weight $(n, m) \in \mathrm{W}_{k, l}$, we write the one-instanton connection $B_{(n)}=B_{n, m}$ in the $(n+1)$-dimensional irreducible representation of $\mathrm{SU}(2)$ as

$$
\begin{aligned}
& B_{n, m}:=B^{11} H_{\alpha_{1}}+B^{12} E_{\alpha_{1}}-\left(B^{12} E_{\alpha_{1}}\right)^{\dagger} \\
& =\sum_{q \in Q_{n}}\left(\left.q B^{11}\right|_{q} ^{n}, m\right\rangle\left\langle{ }_{q}^{n}, m\left|+\frac{1}{2} B^{12} \sqrt{(n-q)(n+q+2)}\right| \stackrel{n}{q+2}, m\right\rangle\left\langle\begin{array}{l}
n \\
q
\end{array}, m\right| \\
& \left.-\frac{1}{2} \overline{B^{12}} \sqrt{(n+q)(n-q+2)}\left|{ }_{q-2}^{n}, m\right\rangle\langle\stackrel{n}{q}, m|\right)
\end{aligned}
$$

where $Q_{n}:=\{-n,-n+2, \ldots, n-2, n\}$, and $B^{i j}$ are the matrix elements of the su(2)-valued instanton connection $B_{(1)}=B-\frac{1}{2} a \mathbf{1}_{2}$. The monopole potential is represented in this basis by $\frac{1}{2} a H_{\alpha_{2}}$. Denote by

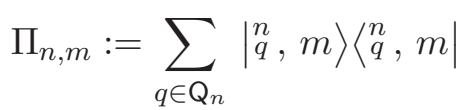

the projection of $\left.\underline{C}^{k, l}\right|_{H}$ onto the irreducible representation $\underline{(n, m)}$ of $H=\mathrm{SU}(2) \times \mathrm{U}(1)$. We further write

$$
\bar{\beta}^{ \pm}=\bar{\beta}^{1} E_{\alpha_{1}+\alpha_{2}}^{ \pm}+\bar{\beta}^{2} E_{\alpha_{2}}^{ \pm}=\sum_{(n, m) \in \mathrm{W}_{k, l}} \bar{\beta}_{n, m}^{ \pm}
$$

where

$$
\begin{aligned}
& \bar{\beta}_{n, m}^{ \pm}:=\frac{\Lambda_{k, l}^{ \pm}(n, m)}{\sqrt{2(n+1)}} \sum_{q \in Q_{n}}\left(\sqrt{n \pm q+1 \pm 1} \bar{\beta}^{1}\left|\begin{array}{l}
n \pm 1 \\
q+1
\end{array}, m+3\right\rangle\left\langle{ }_{q}^{n}, m\right|\right. \\
& \left.+\sqrt{n \mp q+1 \pm 1} \bar{\beta}^{2}\left|\begin{array}{l}
n \pm 1 \\
q-1
\end{array}, m+3\right\rangle\left\langle{ }_{q}^{n}, m\right|\right)
\end{aligned}
$$


are the $(n \pm 1+1) \times(n+1)$ matrix blocks of $G$-equivariant elementary bundle morphisms between $\mathcal{H}_{n, m}$ and $\mathcal{H}_{n \pm 1, m+3}$, together with their hermitean conjugates $\beta_{n, m}^{ \pm}:=\bar{\beta}_{n, m}^{ \pm}{ }^{\dagger}$.

By introducing the projection $\pi_{n, m}$ onto the sub-bundle $E_{n, m} \rightarrow M$, the anti-hermitean $G$ equivariant gauge connection $\mathcal{A}$ on the bundle (2.19) over $M \times \mathbb{C} P^{2}$ can be written as

$$
\begin{aligned}
\mathcal{A}=\sum_{(n, m) \in \mathrm{W}_{k, l}}\left(A^{n, m} \otimes\right. & \Pi_{n, m}+\pi_{n, m} \otimes\left(B_{n, m}+\frac{m}{2} a \Pi_{n, m}\right) \\
& \left.+\phi_{n, m}^{+} \otimes \bar{\beta}_{n, m}^{+}+\phi_{n, m}^{-} \otimes \bar{\beta}_{n, m}^{-}-\phi_{n, m}^{+}{ }^{\dagger} \otimes \beta_{n, m}^{+}-\phi_{n, m}^{-}{ }^{\dagger} \otimes \beta_{n, m}^{-}\right) .
\end{aligned}
$$

Note that when $j_{+}=\frac{k}{2}$, one has $\Lambda_{k, l}^{+}(n, m)=0$ for all $j_{-}$and when $j_{-}=0$, one has $\Lambda_{k, l}^{-}(n, m)=0$ for all $j_{+}$, so the corresponding fields $\bar{\beta}_{n, m}^{ \pm}$and $\phi_{n, m}^{ \pm}$also vanish. These two cases correspond respectively to the rightmost and bottom edges in (2.11). We can thus associate the Higgs fields $\phi_{n, m}^{+}$with the horizontal links in (2.11) and $\phi_{n, m}^{-}$with the vertical links. Then there are a total of $2 k l+k+l$ independent fields $\phi_{n, m}^{ \pm}$.

The matrix elements of the curvature two-form

$$
\mathcal{F}=\mathrm{d} \mathcal{A}+\mathcal{A} \wedge \mathcal{A}
$$

are straightforwardly computed in the Biedenharn basis by using (2.30)-(2.32) [12]. For each weight $(n, m) \in \mathrm{W}_{k, l}$ one finds the diagonal matrix elements

$$
\begin{aligned}
\mathcal{F}^{n, m ; n, m}= & F^{n, m} \otimes \mathbf{1}_{n+1}+\left(\mathbf{1}_{p_{n, m}}-\phi_{n, m}^{+}{ }^{\dagger} \phi_{n, m}^{+}\right) \otimes\left(\beta_{n, m}^{+} \wedge \bar{\beta}_{n, m}^{+}\right) \\
& +\left(\mathbf{1}_{p_{n, m}}-{\phi_{n, m}^{-}}^{\dagger} \phi_{n, m}^{-}\right) \otimes\left({\beta_{n, m}^{-}}^{-} \wedge \bar{\beta}_{n, m}^{-}\right) \\
& +\left(\mathbf{1}_{p_{n, m}}-\phi_{n-1, m-3}^{+} \phi_{n-1, m-3}^{+}\right) \otimes\left(\bar{\beta}_{n-1, m-3}^{+} \wedge \beta_{n-1, m-3}^{+}\right) \\
& +\left(\mathbf{1}_{p_{n, m}}-\phi_{n+1, m-3}^{-} \phi_{n+1, m-3}^{-}\right) \otimes\left(\bar{\beta}_{n+1, m-3}^{-} \wedge \beta_{n+1, m-3}^{-}\right)
\end{aligned}
$$

where $F^{n, m}=\mathrm{d} A^{n, m}+A^{n, m} \wedge A^{n, m}$ is the curvature of the vector bundle $E_{n, m} \rightarrow M$, while the non-vanishing off-diagonal matrix elements are given by

$$
\mathcal{F}^{n \pm 1, m+3 ; n, m}=D \phi_{n, m}^{ \pm} \wedge \bar{\beta}_{n, m}^{ \pm}:=\left(\mathrm{d} \phi_{n, m}^{ \pm}+A^{n \pm 1, m+3} \phi_{n, m}^{ \pm}-\phi_{n, m}^{ \pm} A^{n, m}\right) \wedge \bar{\beta}_{n, m}^{ \pm}
$$

and

$$
\begin{aligned}
& \mathcal{F}^{n+1, m+3 ; n+1, m-3}=\left(\phi_{n, m}^{+} \phi_{n+1, m-3}^{-}-\phi_{n+2, m}^{-} \phi_{n+1, m-3}^{+}\right) \otimes\left(\bar{\beta}_{n, m}^{+} \wedge \bar{\beta}_{n+1, m-3}^{-}\right), \\
& \mathcal{F}^{n+1, m+3 ; n-1, m+3}=\left(\phi_{n, m}^{+} \phi_{n, m}^{-}{ }^{\dagger}-\phi_{n+1, m+3}^{-}{ }^{\dagger} \phi_{n-1, m+3}^{+}\right) \otimes\left(\bar{\beta}_{n, m}^{+} \wedge \beta_{n, m}^{-}\right)
\end{aligned}
$$

along with their hermitean conjugates $\mathcal{F}^{r, s ; n, m}=-\left(\mathcal{F}^{n, m ; r, s}\right)^{\dagger}$ for $(r, s) \neq(n, m)$. The matrix elements (2.49) define bi-fundamental covariant derivatives $D \phi_{n, m}^{ \pm}$of the Higgs fields. The matrix one-form products appearing above are written out explicitly in $₫ \mathrm{~B}$.

\subsection{Invariant spinor fields}

Let $M$ be a complex manifold, so that $d=\operatorname{dim}_{\mathbb{R}}(M)$ is even, and let $K=\bigwedge_{\mathbb{C}}^{d / 2}\left(T^{*} M\right)$ be its canonical line bundle. If $c_{1}(K)=0 \bmod 2$ then $M$ is a spin manifold, while generically $K$ determines a canonical $\operatorname{spin}^{c}$-structure on $M$. The corresponding $\operatorname{spin}^{c}$-bundles are denoted $\triangleq(M) \cong \bigwedge^{0, \bullet}(T M)$ and are obtained by twisting the usual spinor bundles associated to the principal $\operatorname{Spin}(d)$-bundle $P_{\text {Spin }(d)} \rightarrow M$ by $K^{-1 / 2}$. By spinor fields on $M$ or $\mathcal{M}$ we shall always refer to sections of such spin ${ }^{c}$-bundles. 
The equivariant dimensional reduction of massless Dirac spinors on $M \times \mathbb{C} P^{2}$ is defined with respect to (twisted) symmetric fermions on $M$. They act as intertwining operators connecting induced representations of the holonomy group $H=\mathrm{SU}(2) \times \mathrm{U}(1)$ in the $\mathrm{U}(p)$ gauge group, and also in the twisted spinor module $\underline{\Delta}(M)$ which admits the isotopical decomposition

$$
\underline{\Delta}(M)=\bigoplus_{(n, m) \in \mathrm{W}_{k, l}} \Delta_{n, m} \otimes \underline{(n, m)} \quad \text { with } \quad \Delta_{n, m}=\operatorname{Hom}_{H}(\underline{(n, m)}, \underline{\Delta}(M))
$$

obtained by restricting $\triangleq(M)$ to representations of $H \subset \operatorname{Spin}^{c}(d)=\operatorname{Spin}(d) \times_{\mathbb{Z}_{2}} \mathrm{U}(1)$. By Frobenius reciprocity, the multiplicity spaces may be identified as

$$
\Delta_{n, m}=\left(\underline{\Delta}(M)^{\vee} \otimes \Omega^{0}\left(\mathcal{H}_{n, m}\right)\right)^{G}
$$

and hence the isotopical decomposition (2.52) is realized explicitly by constructing symmetric fermions on $M$ as $\mathrm{SU}(3)$-invariant spinors on $M \times \mathbb{C} P^{2}$. They are associated with the eigenspinors of the twisted Dirac operator on $\mathbb{C} P^{2}$, which we describe in some detail.

There is a global obstruction to defining spinors on $\mathbb{C} P^{2}$, but a $\operatorname{spin}^{c}$ structure can be defined by twisting the usual spinor bundle with half-integer powers of the monopole line bundle $\mathcal{L}$. At the level of the twisted Dirac operator, this can be achieved by changing the coupling to the U(1) component of the invariant gauge potential (2.46), and we therefore propose this as a method for describing spinors globally on $\mathbb{C} P^{2}$. The complete spectrum of the Dirac operator on $\mathbb{C} P^{2}$, coupled to arbitrary instanton and monopole backgrounds, was worked out in [16]. The eigenspinors for an arbitrary monopole background, without instantons, were constructed in the context of the fuzzy projective plane $\mathbb{C} P_{F}^{2}$ in [17, 1] while the number of zero modes in a rank two instanton background with arbitrary monopole charge was originally computed in [3]. The number of spinor harmonics in a generic instanton background and with arbitrary monopole number is computed in $\sqrt{\mathrm{C}}$. In this section we will restrict attention to zero modes of the Dirac operator on $\mathbb{C} P^{2}$.

Recall that the pairs $(n, m) \in \mathrm{W}_{k, l}$ appearing in (2.46) have the same even/odd integer parity. Suppose we try to write down a Dirac operator acting on spinors on $M \times \mathbb{C} P^{2}$ coupled to the gauge connection (2.46), transforming under some fixed representation $\rho$ of the subgroup $\prod_{(n, m) \in \mathrm{W}_{k, l}} \mathrm{SU}\left(p_{n, m}\right)$ of the gauge group and under the same weights of $\mathrm{U}(2)$ as those occurring in the decomposition (2.46). Such spinors couple to topologically non-trivial $\mathrm{SU}(2) \times \mathrm{U}(1)$ gauge potentials on $\mathbb{C} P^{2}$. Then there will be an inconsistency because the index of the Dirac operator is fractional, reflecting the fact that spinor fields are never globally well-defined on $\mathbb{C} P^{2}$ in these backgrounds. For a generic $\mathrm{SU}(2) \times \mathrm{U}(1)$ representation $\underline{(n, m)}$, the index is calculated in $₫ \mathrm{C}$ to be

$$
\nu_{b ; n}=\frac{1}{2}(n+1)(b+1)(n+b+2)
$$

where $b=\frac{m-n-3}{2}$. If $n$ and $m$ have the same parity then $b$ is not an integer.

To avoid this obstruction, we modify the Dirac operator by twisting it with a half-integer power $\mathcal{L}_{\widetilde{c}}, \widetilde{c} \in \mathbb{Z}+\frac{1}{2}$ of the monopole line bundle $\mathcal{L}$. The Dirac operator acting on four-component twisted spinor fields $\chi_{n, m ; \widetilde{c}} \in \Omega^{0, \bullet}\left(\mathcal{H}_{n, m+2 \widetilde{c}}\right)$ on $\mathbb{C} P^{2}$, coupled to the rank $n+1$ instanton connection and magnetic monopole potential of charge $\frac{m}{2}+\widetilde{c}$, is then

$$
\not D_{\mathbb{C} P^{2}}=\sum_{(n, m) \in \mathrm{W}_{k, l}}\left(\not_{\mathbb{C} P^{2}} \Pi_{n, m}+\not B_{n, m}+\left(\frac{m}{2}+\widetilde{c}\right) \not k \Pi_{n, m}\right),
$$

where $\not_{\mathbb{C} P^{2}}$ is the naive Dirac operator on $\mathbb{C} P^{2}$ involving only the spin connection. The index for weight $(n, m)$ is again given by (2.54), but now this is an integer when $b=\widetilde{c}+\frac{m-n-3}{2}$. Since $b$

\footnotetext{
${ }^{1}$ Monopole line bundles on $\mathbb{C} P_{F}^{2}$ are also discussed in [18].
} 
depends only on $(n, m)$, and on $\widetilde{c}$ which is half-integer, we denote it by $b_{n, m+c}$ where $c=2 \widetilde{c}$ is an odd integer. Then the index for a given irreducible $\mathrm{U}(2)$-module $\underline{(n, m)}$ is

$$
\nu_{n, m}=\frac{1}{2}(n+1)\left(b_{n, m+c}+1\right)\left(n+b_{n, m+c}+2\right) \text {. }
$$

For fixed $\widetilde{c}$ we shall denote the positive/negative chirality zero modes of the Dirac operator (2.55) by $\chi_{n, m}^{ \pm} \in \mathbb{C}^{2}$. From the explicit construction in [17, it is known that for $n=0$ the index coincides with the total number of zero modes, so either all spinor harmonics have positive chirality or all have negative chirality. We will assume that the same property is true for all $n \geq 1$. Although we do not have a rigorous proof, this seems plausible given the natural identification of the virtual zero mode eigenspaces of $D_{\mathbb{C} P^{2}}$ with irreducible representations of $\mathrm{SU}(3)$ discussed in $\sqrt{\mathrm{C}}$ With this assumption, in a suitable basis there are chiral decompositions

$$
\not D_{\mathbb{C} P^{2}}=\sum_{(n, m) \in \mathrm{W}_{k, l}}\left(\begin{array}{cc}
0 & \not_{n, m}^{+} \\
\not D_{n, m}^{-} & 0
\end{array}\right)
$$

of (2.55) into twisted Dolbeault-Dirac operators $\not_{n, m}^{ \pm}$, such that the index (2.56) is the virtual dimension of the vector space $\operatorname{ker}\left(\not_{n, m}^{+}\right) \ominus \operatorname{ker}\left(\not D_{n, m}^{-}\right)$. Then $\chi_{n, m}^{ \pm} \neq 0$ only when $(n, m) \in \mathrm{W}_{k, l}^{ \pm}$, where

$$
\mathbf{W}_{k, l}^{ \pm}:=\left\{(n, m) \in \mathbf{W}_{k, l} \mid \pm \nu_{n, m}>0\right\} .
$$

We fix a basis of chiral/antichiral spinor harmonics $\chi_{n, m ; \ell}^{ \pm} \in \operatorname{ker}\left(\not_{n, m}^{ \pm}\right), \ell=1, \ldots,\left|\nu_{n, m}\right|$ for each weight $(n, m) \in \mathrm{W}_{k, l}^{ \pm}$. They transform for each $\ell$ in the $(n+1)$-dimensional irreducible representation of the isospin subgroup $\mathrm{SU}(2) \subset H$ of the holonomy group.

We can now use $\left(\underset{\widetilde{2}}{(2.53)}\right.$ to take tensor products of the Dirac zero modes on $\mathbb{C} P^{2}$ with (twisted) Dirac spinors $\psi_{n, m ; \ell}, \widetilde{\psi}_{n, m ; \ell} \in \Omega^{0, \bullet}\left(\rho\left(E_{n, m}\right)\right), \ell=1, \ldots,\left|\nu_{n, m}\right|$ on $M$ to produce fermion fields

$$
\begin{aligned}
& \Psi_{n, m}^{+}=\sum_{\ell=1}^{\nu_{n, m}} \psi_{n, m ; \ell} \otimes \chi_{n, m ; \ell}^{+} \quad \text { and } \quad \Psi_{n, m}^{-}=0 \quad \text { for } \quad(n, m) \in \mathrm{W}_{k, l}^{+}, \\
& \Psi_{n, m}^{-}=\sum_{\ell=1}^{\left|\nu_{n, m}\right|} \widetilde{\psi}_{n, m ; \ell} \otimes \chi_{n, m ; \ell}^{-} \quad \text { and } \quad \Psi_{n, m}^{+}=0 \quad \text { for } \quad(n, m) \in \mathbf{W}_{k, l}^{-} .
\end{aligned}
$$

Note that the spinors $\Psi_{n, m}^{ \pm}$are not chiral on $M \times \mathbb{C} P^{2}$. From these fields we construct a $G$ equivariant Dirac spinor field on $\mathcal{M}=M \times \mathbb{C} P^{2}$ as

$$
\Psi=\left(\begin{array}{l}
\Psi^{+} \\
\Psi^{-}
\end{array}\right)=\bigoplus_{(n, m) \in \mathrm{W}_{k, l}}\left(\begin{array}{l}
\Psi_{n, m}^{+} \\
\Psi_{n, m}^{-}
\end{array}\right)
$$

\section{Quiver gauge theory}

In this section we shall work out the equivariant dimensional reduction of pure massless Yang-MillsDirac theory on the manifold (2.13). We will emphasise the roles played by the SU(2)-instanton and $\mathrm{U}(1)$-monopole background fields on $\mathbb{C} P^{2}$, particularly how they affect the vacuum structure of the quiver gauge theory corresponding to (2.11). We shall also compare the induced equivariant gauge theory on $M$ with that obtained via dimensional reduction over $\mathbb{C} P^{1}[10]$.

\footnotetext{
${ }^{2}$ In any case, if this is not true then the same qualitative conclusions below will hold, but the notation would have to be modified to incorporate the extra spinor harmonics.
} 


\subsection{Reduction of the Yang-Mills action}

We endow the manifold $M$ with local real coordinates $x=\left(x^{\mu}\right) \in \mathbb{R}^{d}$, where the indices $\mu, \nu, \ldots$ run through $1, \ldots, d$. The metric

$$
\mathrm{d} s^{2}=\mathcal{G}_{A B} \mathrm{~d} x^{A} \otimes \mathrm{d} x^{B}
$$

on $\mathcal{M}=M \times \mathbb{C} P^{2}$ will be taken to be the direct product of a chosen riemannian metric on $M$ and the canonical SU(3)-symmetric Kähler metric on $\mathbb{C} P^{2}$ corresponding to the two-form (2.27), where the indices $A, B, \ldots$ run over $1, \ldots, d+4$. Working in the basis $\beta^{i}, \bar{\beta}^{i}$ of invariant forms on $\mathbb{C} P^{2}$ and in the coordinates above, it takes the form

$$
\mathrm{d} s^{2}=G_{\mu \nu} \mathrm{d} x^{\mu} \otimes \mathrm{d} x^{\nu}+2 R^{2}\left(\beta^{1} \otimes \bar{\beta}^{1}+\beta^{2} \otimes \bar{\beta}^{2}\right) .
$$

The line element (3.2) has mass dimension -2 .

The pure Yang-Mills lagrangian on $\mathcal{M}=M \times \mathbb{C} P^{2}$ is given by

$$
\begin{aligned}
L_{\mathrm{YM}}^{k, l}= & -\frac{1}{4 \tilde{g}^{2}} \sqrt{|\mathcal{G}|} \operatorname{tr}_{p \times p} \mathcal{F}_{A B} \mathcal{F}^{A B} \\
= & -\frac{1}{4 \tilde{g}^{2}} \sqrt{|\mathcal{G}|} \operatorname{tr}_{p \times p}\left[\mathcal{F}_{\mu \nu} \mathcal{F}^{\mu \nu}+\frac{1}{2 R^{2}} G^{\mu \nu}\left(\mathcal{F}_{\mu i} \mathcal{F}_{\nu \bar{\imath}}+\mathcal{F}_{\mu \bar{\imath}} \mathcal{F}_{\nu i}\right)\right. \\
& \left.\quad-\frac{1}{2 R^{4}}\left(\left|\mathcal{F}_{1 \overline{1}}\right|^{2}+\left|\mathcal{F}_{2 \overline{2}}\right|^{2}+2\left|\mathcal{F}_{1 \overline{2}}\right|^{2}+2\left|\mathcal{F}_{12}\right|^{2}\right)\right]
\end{aligned}
$$

where we use the matrix notation $|\mathcal{F}|^{2}:=\frac{1}{2}\left(\mathcal{F}^{\dagger} \mathcal{F}+\mathcal{F} \mathcal{F}^{\dagger}\right)$, and $i=1,2$ labels components along $\mathbb{C} P^{2}$ in the basis used in (3.2). The $(d+4)$-dimensional $\mathrm{U}(p)$ Yang-Mills coupling constant $\tilde{g}$ has the standard mass dimension $-\frac{d}{2}$ in order to make (3.3) dimensionless. We substitute (2.48)-(2.51) into (3.3), and take the trace over the representation space $(n, m)$ for each weight $(n, m) \in \mathbf{W}_{k, l}$ making

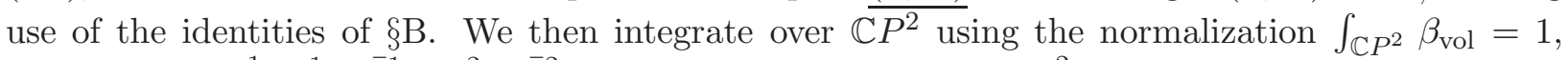
where $\beta_{\mathrm{vol}}:=\frac{1}{2 \pi^{2}} \beta^{1} \wedge \bar{\beta}^{1} \wedge \beta^{2} \wedge \bar{\beta}^{2}$ is the unit volume form of $\mathbb{C} P^{2}$.

After some calculation and rescaling $\phi_{n, m}^{ \pm} \rightarrow \Lambda_{k, l}^{ \pm}(n, m)^{-1} \phi_{n, m}^{ \pm}$, one finds that the dimensional reduction of the corresponding Yang-Mills action

$$
S_{\mathrm{YM}}^{k, l}:=\int_{M \times \mathbb{C} P^{2}} \mathrm{~d}^{d+4} x L_{\mathrm{YM}}^{k, l}
$$


is given by

$$
\begin{aligned}
S_{\mathrm{YM}}^{k, l}= & \frac{\left(\pi R^{2}\right)^{2}}{2 \tilde{g}^{2}} \int_{M} \mathrm{~d}^{d} x \sqrt{|G|} \sum_{(n, m) \in \mathrm{W}_{k, l}} \operatorname{tr}_{p_{n, m} \times p_{n, m}}\left[(n+1)\left(F^{n, m}\right)_{\mu \nu}^{\dagger}\left(F^{n, m}\right)^{\mu \nu}\right. \\
& +\frac{n+2}{2 R^{2}}\left(D_{\mu} \phi_{n, m}^{+}\right)^{\dagger}\left(D^{\mu} \phi_{n, m}^{+}\right)+\frac{n+1}{2 R^{2}}\left(D_{\mu} \phi_{n-1, m-3}^{+}\right)\left(D^{\mu} \phi_{n-1, m-3}^{+}\right)^{\dagger} \\
& +\frac{n}{2 R^{2}}\left(D_{\mu} \phi_{n, m}^{-}\right)^{\dagger}\left(D^{\mu} \phi_{n, m}^{-}\right)+\frac{n+1}{2 R^{2}}\left(D_{\mu} \phi_{n+1, m-3}^{-}\right)\left(D^{\mu} \phi_{n+1, m-3}^{-}\right)^{\dagger} \\
& +\frac{n+2}{2 R^{4}}\left(\Lambda_{k, l}^{+}(n, m)^{2} \mathbf{1}_{p_{n, m}}-\phi_{n, m}^{+} \phi_{n, m}^{+}\right)^{2}+\frac{n}{2 R^{4}}\left(\Lambda_{k, l}^{-}(n, m)^{2} \mathbf{1}_{p_{n, m}}-\phi_{n, m}^{-}{ }^{\dagger} \phi_{n, m}^{-}\right)^{2} \\
& +\frac{(n+1)^{2}}{2 n R^{4}}\left(\Lambda_{k, l}^{+}(n-1, m-3)^{2} \mathbf{1}_{p_{n, m}}-\phi_{n-1, m-3}^{+} \phi_{n-1, m-3}^{+}\right)^{2} \\
& +\frac{(n+1)^{2}}{2(n+2) R^{4}}\left(\Lambda_{k, l}^{-}(n+1, m-3)^{2} \mathbf{1}_{p_{n, m}}-\phi_{n+1, m-3}^{-} \phi_{n+1, m-3}^{-}\right)^{2} \\
& +\frac{n(n+2)}{2(n+1) R^{4}} \mid \phi_{n, m}^{+} \phi_{n, m}^{-}{ }^{\dagger}-\frac{\Lambda_{k, l}^{+}(n, m) \Lambda_{k, l}^{-}(n, m)}{\Lambda_{k, l}^{+}(n-1, m+3) \Lambda_{k, l}^{-}(n+1, m+3)} \phi_{n+1, m+3}^{-}\left(\left.\phi_{n-1, m+3}^{+}\right|^{2}\right. \\
& \left.+\frac{n+3}{6 R^{4}}\left|\phi_{n, m}^{+} \phi_{n+1, m-3}^{-}-\frac{\Lambda_{k, l}^{+}(n, m) \Lambda_{k, l}^{-}(n+1, m-3)}{\Lambda_{k, l}^{+}(n+1, m-3) \Lambda_{k, l}^{-}(n+2, m)} \phi_{n+2, m}^{-} \phi_{n+1, m-3}^{+}\right|^{2}\right] .
\end{aligned}
$$

From (2.49) it follows that the $\mathrm{U}(1)$ factor in the structure group $\mathrm{U}(p) \cong \mathrm{U}(1) \times \mathrm{SU}(p)$ does not enter the bicovariant derivatives of the rectangular scalar fields $\phi_{n, m}^{ \pm}$. We can therefore restrict to gauge group $\mathrm{SU}(p)$, and the decomposition (2.17) is then modified to

$$
\mathrm{SU}(p) \longrightarrow \mathrm{U}(1)^{(k+1)(l+1)-1} \times \prod_{(n, m) \in \mathrm{W}_{k, l}} \mathrm{SU}\left(p_{n, m}\right) \quad \text { with } \quad \sum_{(n, m) \in \mathrm{W}_{k, l}}(n+1) p_{n, m}=p
$$

where $(k+1)(l+1)$ is the number of elements of the weight set $\mathbf{W}_{k, l}$.

The gauge coupling in $d$ dimensions should have mass dimension $2-\frac{d}{2}$, and therefore we define $g^{2}=\tilde{g}^{2} / 2\left(\pi R^{2}\right)^{2}$ as the $d$-dimensional gauge coupling constant. We then rescale

$$
\phi_{n, m}^{ \pm} \longrightarrow \frac{2 g R}{\sqrt{n+1 \pm 1}} \phi_{n, m}^{ \pm} \quad \text { and } \quad A^{n, m} \longrightarrow \frac{g}{\sqrt{n+1}} A^{n, m}
$$

so that the scalar and gauge fields have the correct canonical dimensions and kinetic term normalizations for a $d$-dimensional field theory (with dimensionless coordinates). The Higgs potential in the scalar fields $\phi_{n, m}^{ \pm}$in (3.5) generically leads to spontaneous symmetry breaking, as a direct consequence of the non-trivial background instanton and monopole charges on $\mathbb{C} P^{2}$. Since this potential is a sum of non-negative terms, it is easy to write down the general structure of the vacua in the Higgs sector of the field theory. In particular, they obey the equations

$$
\begin{aligned}
\phi_{n, m}^{ \pm}{ }^{\dagger} \phi_{n, m}^{ \pm} & =\frac{(n+1 \pm 1) \Lambda_{k, l}^{ \pm}(n, m)^{2}}{4 g^{2} R^{2}} \mathbf{1}_{p_{n, m}}, \\
\phi_{n, m}^{ \pm} \phi_{n, m}^{ \pm}{ }^{\dagger} & =\frac{(n+1 \pm 1) \Lambda_{k, l}^{ \pm}(n, m)^{2}}{4 g^{2} R^{2}} \mathbf{1}_{p_{n \pm 1, m+3}} .
\end{aligned}
$$

The vanishing of the last two terms in (3.5) represent the relations of the quiver (2.11) [12] and has a natural algebraic meaning in terms of the operators

$$
\begin{array}{r}
\phi^{ \pm}:=\sum_{(n, m) \in \mathrm{W}_{k, l}} \Lambda_{k, l}^{ \pm}(n, m)^{-1} \phi_{n, m}^{ \pm} \otimes\left(\sum _ { q \in \mathrm { Q } _ { n } } \left(\sqrt{n \pm q+1 \pm 1}\left|\begin{array}{l}
n \pm 1 \\
q+1
\end{array}, m+3\right\rangle\left\langle\begin{array}{l}
n \\
q
\end{array}, m\right|\right.\right. \\
\left.+\sqrt{n \mp q+1 \pm 1}\left|\begin{array}{l}
n \pm 1 \\
q-1
\end{array}, m+3\right\rangle\left\langle\left\langle\begin{array}{l}
n \\
q
\end{array}, m\right|\right)\right)
\end{array}
$$


defined with respect to the Biedenharn basis of $\$ 2.4$. Then, in addition to (3.8), the Higgs vacua are determined by the matrix commutativity relations

$$
\left[\boldsymbol{\phi}^{+}, \boldsymbol{\phi}^{-}\right]=0 \quad \text { and } \quad\left[\boldsymbol{\phi}^{+}, \boldsymbol{\phi}^{-\dagger}\right]=0 .
$$

When $p_{n, m}=r$ for all weights $(n, m) \in \mathrm{W}_{k, l}$, the gauge symmetry reduction is given by

$$
\mathrm{SU}(p) \longrightarrow \mathrm{U}(1)^{(k+1)(l+1)-1} \times \mathrm{SU}(r)^{(k+1)(l+1)} \quad \text { with } \quad p=r d^{k, l}
$$

where $d^{k, l}$ are the dimensions (2.4). In this special case an explicit solution of (3.8) is given by $\phi_{n, m}^{ \pm}=\phi_{n, m}^{ \pm}{ }^{0}$, where

$$
\phi_{n, m}^{ \pm}{ }^{0}=\frac{\sqrt{n+1 \pm 1} \Lambda_{k, l}^{ \pm}(n, m)}{2 g R} U_{n, m}^{ \pm}
$$

for $(n, m) \in \mathrm{W}_{k, l}$. This solution involves $2 k l+k+l$ unitary degrees of freedom $U_{n, m}^{ \pm} \in \mathrm{U}(r)$, one for each Higgs field $\phi_{n, m}^{ \pm}$. We can associate each such unitary group element with a link of the lattice (2.11), which defines a gauge field on the quiver lattice. However, they are not all independent, because the commutation relations (3.10) require that they obey

$$
U_{n+1, m+3}^{-} U_{n, m}^{+}=U_{n-1, m+3}^{+} U_{n, m}^{-},
$$

which is equivalent to requiring that their oriented product around the four links of any plaquette in the quiver lattice must be equal to unity. Thus the Higgs vacua correspond to flat connections of lattice gauge theory on the finite quiver lattice. However, there is no vacuum moduli space, because we can set $k l+k+l$ of these unitary degrees of freedom to the identity using a gauge transformation in the $\mathrm{U}(r)^{(k+1)(l+1)-1}$ subgroup of (3.11), and then eliminate the remaining ones using the $k l$ plaquette relations (3.13). Thus the solution (3.12) breaks the gauge symmetry of the $d$-dimensional field theory on $M$ to the diagonal $\mathrm{SU}(r)$ subgroup, leaving $(k l+k+l) r^{2}$ massive gauge bosons and $(3 k l+k+l) r^{2}$ physical Higgs fields. This mechanism induces physical masses proportional to $\frac{1}{R}$. In subsequent sections we shall work out some explicit examples.

\subsection{Reduction of the Dirac action}

To describe the form of the fermionic action for the invariant spinor fields constructed in 2.5 , we first need to set up some Clifford algebra notation. The left-invariant one-forms defined in (2.22) are proportional to orthonormal one-forms on $\mathbb{C} P^{2}$ and they define vierbeins $e^{i}{ }_{a}$ on $\mathbb{C} P^{2}$ through

$$
\beta^{i}=\frac{1}{R} e_{a}^{i} \mathrm{~d} y^{a} \quad \text { and } \quad \bar{\beta}^{i}=\frac{1}{R} e^{\bar{a}} \overline{\mathrm{d}} \bar{y}^{a},
$$

where $i=1,2$ is an orthonormal index and $a=1,2$ is a coordinate index. With $M$ a complex manifold as in $\$ 2.5$, the generators of the Clifford algebra $\mathrm{C} \ell\left(M \times \mathbb{C} P^{2}\right)$ obey

$$
\Gamma^{A} \Gamma^{B}+\Gamma^{B} \Gamma^{A}=-2 \mathcal{G}^{A B} \mathbf{1}_{2^{d / 2+2}} \quad \text { with } A, B=1, \ldots, d+4 .
$$

The gamma-matrices in (3.15) may be decomposed as

$$
\left\{\Gamma^{A}\right\}=\left\{\Gamma^{\mu}, \Gamma^{a}, \Gamma^{\bar{a}}\right\} \quad \text { with } \quad \Gamma^{\mu}=\gamma^{\mu} \otimes \mathbf{1}_{4}, \quad \Gamma^{a}=\gamma \otimes \tau^{a} \quad \text { and } \quad \Gamma^{\bar{a}}=\gamma \otimes \tau^{\bar{a}}
$$

where our convention is $\Gamma^{a} \Gamma^{\bar{a}}+\Gamma^{\bar{a}} \Gamma^{a}=-\mathcal{G}^{a \bar{a}} \mathbf{1}_{2^{d / 2+2}}$ in complex coordinates. The $2^{d / 2} \times 2^{d / 2}$ matrices $\gamma^{\mu}=-\left(\gamma^{\mu}\right)^{\dagger}$ act locally on the twisted spinor module $\Delta(M)$ over the Clifford algebra bundle $\mathrm{C} \ell(M) \rightarrow M$ with the relations

$$
\gamma^{\mu} \gamma^{\nu}+\gamma^{\nu} \gamma^{\mu}=-2 G^{\mu \nu} \mathbf{1}_{2^{d / 2}} \quad \text { with } \quad \mu, \nu=1, \ldots, d,
$$


while

$$
\gamma=\frac{\mathrm{i}^{d / 2} \sqrt{G}}{d !} \epsilon_{\mu_{1} \cdots \mu_{d}} \gamma^{\mu_{1}} \cdots \gamma^{\mu_{d}} \quad \text { with } \quad(\gamma)^{2}=\mathbf{1}_{2^{d / 2}} \quad \text { and } \quad \gamma \gamma^{\mu}=-\gamma^{\mu} \gamma
$$

is the corresponding chirality operator. Here $\epsilon_{\mu_{1} \ldots \mu_{d}}$ is the Levi-Civita symbol with $\epsilon_{12 \cdots d}=+1$.

The coordinate basis gamma-matrices $\tau^{a}$ and $\tau^{\bar{a}}$ on $\mathbb{C} P^{2}$ are related to their orthonormal counterparts by

$$
\sigma^{i}=\mathrm{i} e_{a}^{i} \tau^{a} \quad \text { and } \quad \sigma^{\bar{\imath}}=-\mathrm{i} e^{\bar{\imath}} \bar{a} \tau^{\bar{a}}
$$

with the normalisation chosen so that

$$
\sigma^{i} \sigma^{\bar{\jmath}}+\sigma^{\bar{\jmath}} \sigma^{i}=\delta^{i j} \mathbf{1}_{4}
$$

It is a standard construction [19] to choose a basis in which $\left(\sigma^{i}\right)^{2}=\left(\sigma^{\bar{\imath}}\right)^{2}=0$, and to associate $\sigma^{\bar{\imath}}$ and $\sigma^{i}$ respectively with creation and annihilation operators acting on a fermionic Fock space with vacuum vector $|\Omega\rangle$ such that $\sigma^{i}|\Omega\rangle=0$. A general Fock space state

$$
|\chi\rangle:=\chi_{0}(y, \bar{y}) \otimes|\Omega\rangle+\chi_{\bar{\imath}}(y, \bar{y}) \otimes \sigma^{\bar{\imath}}|\Omega\rangle+\frac{1}{2} \chi_{\bar{\imath} \bar{\jmath}}(y, \bar{y}) \otimes \sigma^{\bar{\imath}} \bar{\jmath}|\Omega\rangle,
$$

with $\sigma^{\bar{\imath}} \bar{\jmath}:=\frac{1}{2}\left[\sigma^{\bar{\imath}}, \sigma^{\bar{\jmath}}\right]$, corresponds locally to a Dirac spinor on $\mathbb{C} P^{2}$, though of course it may not extend to a global spinor field. The chirality operator on $\mathbb{C} P^{2}$ is $\widetilde{\sigma}=\left[\sigma^{1}, \sigma^{\overline{1}}\right]\left[\sigma^{2}, \sigma^{\overline{2}}\right]$ and $\chi_{\bar{\imath}}$ are the two components of a negative chirality spinor, while $\chi_{0}$ and $\chi_{\overline{1}} \overline{2}$ are the two components of a positive chirality spinor. In terms of holonomy, $\chi_{\bar{\imath}}$ is a doublet of $\mathrm{SU}(2)$ while $\chi_{0}$ and $\chi_{\overline{1}} \overline{2}$ are both $\mathrm{SU}(2)$-singlets.

An alternative way of understanding these representation assignments, which will be useful in later sections, follows from the general construction in [16. Spinor fields on $\mathbb{C} P^{2}$ transform in the $4 \times 4$ (reducible) spinor representation of $H=\mathrm{SU}(2) \times \mathrm{U}(1)$ given by

$$
\begin{array}{rlrl} 
& \Sigma_{E_{\alpha_{1}}}=\sigma^{1} \sigma^{\overline{2}} & \text { and } & \Sigma_{E_{-\alpha_{1}}}=\Sigma_{E_{\alpha_{1}}}^{\dagger}=\sigma^{2} \sigma^{\overline{1}}, \\
\Sigma_{H_{\alpha_{1}}}=\sigma^{1} \sigma^{\overline{1}}-\sigma^{2} \sigma^{\overline{2}} & \text { and } & \Sigma_{H_{\alpha_{2}}}=\sigma^{1} \sigma^{\overline{1}}+\sigma^{2} \sigma^{\overline{2}}-\mathbf{1}_{4} .
\end{array}
$$

These generators constitute a traceless representation of the $\mathrm{su}(2) \oplus \mathrm{u}(1)$ subalgebra of (2.3), as is easily checked using (3.20). The representation content is revealed by evaluating the second order Casimir invariants of $\mathrm{SU}(2)$ and $\mathrm{U}(1)$ to get

$$
\frac{1}{2}\left|\Sigma_{E_{\alpha_{1}}}\right|^{2}+\frac{1}{2}\left(\Sigma_{H_{\alpha_{1}}}\right)^{2}=\frac{3}{8}\left(\mathbf{1}_{4}-\tilde{\sigma}\right) \quad \text { and } \quad \frac{1}{2}\left(\Sigma_{H_{\alpha_{2}}}\right)^{2}=\frac{1}{8}\left(\mathbf{1}_{4}+\tilde{\sigma}\right) .
$$

It follows that negative chirality spinors live in the representation $(1,0)$, while positive chirality spinors are given by a pair of $\mathrm{SU}(2)$-singlets with opposite hypercharge $Y= \pm 1$ in the $H$-module $\underline{(0, \pm 3)}$.

These states correspond respectively to the instanton bundle $\mathcal{I}$, with fibres transforming under the representation $(1,0)$, and the monopole line bundles $\mathcal{L}_{ \pm 3 / 2}$, with fibres transforming under the representation $\overline{(0, \pm 3)}$. None of these bundles is globally well-defined of course. In order to get well-defined bundles on $\mathbb{C} P^{2}$, we must tensor the would-be spin bundle with non-trivial gauge bundles $\mathcal{I}_{n} \otimes \mathcal{L}_{\widetilde{m} / 2}$ whose fibres transform according to the representation $(n, \widetilde{m})$ of $\mathrm{SU}(2) \times \mathrm{U}(1)$ with $n$ and $\widetilde{m}=m+c$ of opposite even/odd integer parity. These bundles do not exist on their own but, as described in $\$ 2.5$, their tensor product does. The complete $\mathrm{SU}(2) \times \mathrm{U}(1)$ representation content of these bundles is given by the decomposition into irreducible modules




where the square brackets segregate the spinor chiralities.

For each weight $(n, m) \in \mathbf{W}_{k, l}$, the complete spectrum of the twisted Dirac operator on $\mathbb{C} P^{2}$ consists of $4(n+1)$ families of infinite discrete sequences of eigenvalues, one family for each state on the right-hand side of (3.24). The non-zero eigenvalues come in positive and negative pairs giving the $2(n+1)$ sequences listed below. The spectrum therefore grows rapidly more complicated as $n$ increases. Note that at least some of the corresponding eigenspinors must necessarily have different assignments of $\mathrm{SU}(2) \times \mathrm{U}(1)$ quantum numbers for their two chiral components. After dimensional reduction, the eigenspinors on $\mathbb{C} P^{2}$ with non-zero eigenvalues will induce a total of $4 d^{k, l}$ infinite discrete families of fermion fields on $M$ with both a kinetic mass term, given by the Dirac eigenvalues on $\mathbb{C} P^{2}$, and Yukawa couplings.

The eigenvalues and their multiplicities can be read off from the explicit formulas of [16, $\S \mathrm{B}]$. There are $2 n+2$ infinite sequences, with $n+2$ families coming from the states in $\left(n^{\prime}, \widetilde{m}\right)$ with $n^{\prime}=n+1$ and $n$ families coming from the states with $n^{\prime}=n-1$. Denoting the eigenvalues by $\frac{\lambda_{N}}{R}$ and their degeneracies by $d_{N}$, we distinguish each sequence by $n+2$ integers $\eta_{+}$and $n$ integers $\eta_{-}$ with

$$
\begin{aligned}
& \lambda_{N}^{2}=N(N+n+3)+\eta_{+}^{2}+\frac{\left|\eta_{+}\right|}{2}(2 N+n+3)-\frac{\eta_{+}}{2}|\widetilde{m}|+n+2 \\
& d_{N}=\frac{1}{8}\left(2 N+n+3+\epsilon_{+}\left(4 \eta_{+}-|\widetilde{m}|\right)\right)\left(2 N+n+3-\epsilon_{+}\left(2 \eta_{+}-|\widetilde{m}|\right)\right)\left(2 N+n+3+\left|\eta_{+}\right|\right)
\end{aligned}
$$

for $n^{\prime}=n+1$, and

$$
\begin{aligned}
\lambda_{N}^{2} & =N(N+n+1)+\eta_{-}^{2}+\frac{\left|\eta_{-}\right|}{2}(2 N+n+1)-\frac{\eta_{-}}{2}|\widetilde{m}| \\
d_{N} & =\frac{1}{8}\left(2 N+n+1+\epsilon_{-}\left(4 \eta_{-}-|\widetilde{m}|\right)\right)\left(2 N+n+1-\epsilon_{-}\left(2 \eta_{-}-|\widetilde{m}|\right)\right)\left(2 N+n+1+\left|\eta_{-}\right|\right)
\end{aligned}
$$

for $n^{\prime}=n-1$, where

$$
\eta_{ \pm}=-\frac{1}{2}(n \pm 1+|\widetilde{m}|), \ldots, \frac{1}{2}(n \pm 1+|\widetilde{m}|) .
$$

In both cases $N=0,1, \ldots$, while $\epsilon_{ \pm}=1$ for $\eta_{ \pm} \geq 0$ and $\epsilon_{ \pm}=-1$ for $\eta_{ \pm}<0$. We shall see some explicit examples in the following sections.

We will now construct the $\mathcal{E}^{k, l}$-twisted Dirac operator $\not{D}=\Gamma^{A} \mathcal{D}_{A}$ on $\mathcal{M}=M \times \mathbb{C} P^{2}$, corresponding to the equivariant gauge potential $\mathcal{A}$ in (2.46) and acting on the spinor fields (2.60), in terms of the $\operatorname{spin}^{c}$ Dirac operator (2.55) on $\mathbb{C} P^{2}$ and the $E^{k, l}$-twisted $\operatorname{spin}^{c}$ Dirac operator $\not D=\gamma^{\mu} D_{\mu}$ on $M$. The latter operator is given by

$$
\not D=\sum_{(n, m) \in \mathrm{W}_{k, l}}\left(\not \partial_{M}-\frac{1}{2} \not k+\not A^{n, m}\right) \otimes \pi_{n, m}
$$

where $\not_{M}$ is the naive Dirac operator on $M$ involving only the spin connection on the principal $\mathrm{SO}(d)$-bundle $P_{\mathrm{SO}(d)} \rightarrow M$ and the generators of $\mathrm{SO}(d)$ in the spinor representation, while $\kappa$ is an anti-hermitean connection on the canonical line bundle $K \rightarrow M$. Using (3.14) and (3.19) one then finds

$$
\begin{aligned}
\not D= & \not D \otimes \mathbf{1}_{4}+\gamma \otimes \mathbb{D}_{\mathbb{C} P^{2}} \\
& +\frac{1}{R} \sum_{(n, m) \in \mathrm{W}_{k, l}}\left(\phi_{n, m}^{+} \gamma \otimes \sigma_{n, m}^{+}+\phi_{n, m}^{-} \gamma \otimes \sigma_{n, m}^{-}-\phi_{n, m}^{+}{ }^{\dagger} \gamma \otimes \sigma_{n, m}^{+}{ }^{\dagger}-\phi_{n, m}^{-}{ }^{\dagger} \gamma \otimes \sigma_{n, m}^{-}{ }^{\dagger}\right)
\end{aligned}
$$


where, in complete analogy with (2.44) and (2.45), we have defined

$$
\sigma^{ \pm}=\mathrm{i} \sigma^{\overline{1}} \otimes E_{\alpha_{1}+\alpha_{2}}^{ \pm}+\mathrm{i} \sigma^{\overline{2}} \otimes E_{\alpha_{2}}^{ \pm}=\sum_{(n, m) \in \mathrm{W}_{k, l}} \sigma_{n, m}^{ \pm}
$$

Using the twisted Dirac operator (3.29), we may define an euclidean fermionic action functional on the space of $\mathrm{L}^{2}$-sections (2.60) by

$$
S_{\mathrm{D}}^{k, l}:=\int_{M \times \mathbb{C} P^{2}} \mathrm{~d}^{d+4} x \sqrt{|\mathcal{G}|} \Psi^{\dagger} \not \mathcal{D} \Psi
$$

where $\Psi$ has canonical mass dimension $\frac{1}{2}(d+3)$. In lorentzian signature the adjoint spinor $\Psi^{\dagger}$ should be replaced by $\bar{\Psi}:=\frac{1}{\sqrt{-\mathcal{G}^{00}}} \Psi^{\dagger} \Gamma^{0}$. For definiteness, we shall only consider the case where the spinor field $\Psi$ transforms under the fundamental representation of the initial gauge group $\mathrm{SU}(p)$. Other fermion representations of $\mathrm{SU}(p)$ can be treated similarly. We substitute (2.59) and integrate over $\mathbb{C} P^{2}$ in (3.31). The zero modes of $\not_{\mathbb{C} P^{2}}$ can be chosen to be orthogonal and normalised such that

$$
\int_{\mathbb{C} P^{2}} \chi_{n^{\prime}, m^{\prime} ; \ell^{\prime}}^{\dagger} \chi_{n, m ; \ell}^{ \pm} \beta_{\mathrm{vol}}=\delta_{n, n^{\prime}} \delta_{m, m^{\prime}} \delta_{\ell, \ell^{\prime}} \quad \text { and } \quad \int_{\mathbb{C} P^{2}} \chi_{n^{\prime}, m^{\prime} ; \ell^{\prime}}^{\mp} \chi_{n, m ; \ell}^{ \pm} \beta_{\mathrm{vol}}=0
$$

where the second equality follows from the fact that the sets $\mathrm{W}_{k, l}^{+}$and $\mathrm{W}_{k, l}^{-}$in (2.58) are disjoint. Since $\chi_{n, m ; \ell}^{ \pm}$are spinor harmonics on $\mathbb{C} P^{2}$, one might naively expect that the fermion fields $\psi_{n, m ; \ell}$ and $\widetilde{\psi}_{n, m ; \ell}$ will be massless spinors on $M$. However, the Higgs field terms in (3.29) can give rise to Yukawa couplings and, due to spontaneous symmetry breaking, induce masses of order $\frac{1}{R}$ to the $d$-dimensional spinors. We shall now explain precisely how this comes about.

Recall that the fermion zero modes depend on the twisting parameter $c=2 \widetilde{c}$ introduced in $₫ 2.5$, We will now show how to uniquely fix this free parameter such that the reduction of the action (3.31) generically contains Yukawa couplings. We consider background gauge fields on $\mathbb{C} P^{2}$ for which the index (2.56) takes values $\nu_{n, m}= \pm 1$. The spinor harmonic modes are particularly simple in this case [14]. They arise as a result of the gauge connections of the $\mathrm{SU}(2) \times \mathrm{U}(1)$ gauge theory on $\mathbb{C} P^{2}$ exactly cancelling the spin connection, so that the Dirac operator truncates to the (untwisted) Dolbeault operator on $\mathbb{C} P^{2}$ and the components of the spinors in (3.21) are simply constants. Note that this can only occur when $n=0,1$, and hence by (2.10) for $m=-2(k-l),-2(k-l) \pm 3$. For any given irreducible $\mathrm{SU}(3)$-representation $\underline{C}^{k, l}$, it is easy to deduce from $(2.56)$ that the unique $\operatorname{spin}^{c}$ structure on $\mathbb{C} P^{2}$ accommodating these fields has twisting parameter

$$
c=2(k-l)-3 .
$$

Then the chiral fermion mode with $(n, m)=(0,-2(k-l))$ (and $\left.\nu_{n, m}=+1\right)$ will have a Yukawa coupling to the antichiral mode with $(n, m)=(1,-2(k-l)+3)\left(\right.$ and $\left.\nu_{n, m}=-1\right) 3$

The positive chirality mode with respect to the Biedenharn basis of \$2.4 is given by

$$
\left.\chi_{0,-2(k-l)}^{+}=|\Omega\rangle \otimes||_{0}^{0},-2(k-l)\right\rangle
$$

while the negative chirality mode, which is a doublet of the $\mathrm{SU}(2)$ gauge theory on $\mathbb{C} P^{2}$, is

$$
\left.\chi_{1,-2(k-l)+3}^{-}=\frac{1}{\sqrt{2}}\left(\sigma^{\overline{1}}|\Omega\rangle \otimes||_{1}^{1},-2(k-l)+3\right\rangle+\sigma^{\overline{2}}|\Omega\rangle \otimes|\stackrel{1}{-1},-2(k-l)+3\rangle\right) .
$$

\footnotetext{
${ }^{3}$ For $l \geq 1$, one can alternatively choose $c=2(k-l)+3$, and couple the chiral mode with $(n, m)=(0,-2(k-l))$ to the antichiral mode with $(n, m)=(1,-2(k-l)-3)$.
} 
From the explicit formulas (2.41) one finds $\Lambda_{k, l}^{-}(0,-2(k-l))=\Lambda_{k, l}^{-}(1,-2(k-l)+3)=0$, and consequently the only contributing operator from (3.30) is given by

$\left.\sigma_{0,-2(k-l)}^{+}=\frac{\mathrm{i}}{\sqrt{2}} \sqrt{k(l+2)}\left(\left.\sigma^{\overline{1}} \otimes\right|_{1} ^{1},-2(k-l)+3\right\rangle\left\langle{ }_{0}^{0},-2(k-l)\left|+\sigma^{\overline{2}} \otimes\right| \stackrel{1}{-1},-2(k-l)+3\right\rangle\left\langle{ }_{0}^{0},-2(k-l)\right|\right)$

with

$$
\begin{aligned}
\sigma_{0,-2(k-l)}^{+} \chi_{0,-2(k-l)}^{+} & =\mathrm{i} \sqrt{k(l+2)} \chi_{1,-2(k-l)+3}^{-}, \\
\sigma_{0,-2(k-l)}^{+} \chi_{1,-2(k-l)+3}^{-} & =\mathrm{i} \sqrt{k(l+2)} \chi_{0,-2(k-l)}^{+} .
\end{aligned}
$$

These are then the only surviving contributions from the Higgs field terms in (3.29) after integration over $\mathbb{C} P^{2}$ using (3.32).

We now rescale the bosonic fields as in (3.7) and the fermionic fields as

$$
\psi_{n, m ; \ell} \longrightarrow \frac{1}{\sqrt{2} \pi R^{2}} \psi_{n, m ; \ell} \quad \text { and } \quad \widetilde{\psi}_{n, m ; \ell} \longrightarrow \frac{1}{\sqrt{2} \pi R^{2}} \widetilde{\psi}_{n, m ; \ell}
$$

in order to give all fields the correct canonical dimensions and kinetic term normalizations on $M$. Putting everything together, the dimensional reduction of the Dirac action (3.31) is given by

$$
\begin{aligned}
S_{\mathrm{D}}^{k, l}=\int_{M} \mathrm{~d}^{d} x \sqrt{|G|}[ & \sum_{(n, m) \in \mathrm{W}_{k, l}^{+}} \sum_{\ell=1}^{\nu_{n, m}}\left(\psi_{n, m ; \ell}\right)^{\dagger} \not D\left(\psi_{n, m ; \ell}\right) \\
& +\sum_{(n, m) \in \mathrm{W}_{k, l}^{-}} \sum_{\ell=1}^{\left|\nu_{n, m}\right|}\left(\widetilde{\psi}_{n, m ; \ell}\right)^{\dagger} \not D\left(\widetilde{\psi}_{n, m ; \ell}\right) \\
& +\sqrt{2 k(l+2)} g\left(\left(\psi_{0,-2(k-l)}\right)^{\dagger} \phi_{0,-2(k-l)}^{+}{ }^{\dagger} \psi_{1,-2(k-l)+3}\right. \\
& \left.\left.+\left(\psi_{1,-2(k-l)+3}\right)^{\dagger} \phi_{0,-2(k-l)}^{+} \psi_{0,-2(k-l)}\right)\right]
\end{aligned}
$$

where we have abbreviated $\psi_{0,-2(k-l)}:=\psi_{0,-2(k-l) ; 0}$ and $\psi_{1,-2(k-l)+3}:=\gamma \widetilde{\psi}_{1,-2(k-l)+3 ; 0}$. The fermion fields $\psi_{n, m ; \ell}$ and $\widetilde{\psi}_{n, m ; \ell}$ for each $\ell=1, \ldots,\left|\nu_{n, m}\right|$ transform in the fundamental representation of $\mathrm{SU}\left(p_{n, m}\right)$. The dimensionally reduced field theory thus contains Yukawa interactions for all $k>0$. If the Higgs field $\phi_{0,-2(k-l)}^{+}$acquires a non-zero vacuum expectation value $\phi_{0,-2(k-l)}^{+}$by dynamical symmetry breaking, then the fermion fields $\psi_{0,-2(k-l)}$ and $\psi_{1,-2(k-l)+3}$ acquire a mass matrix. In the special case (3.12), the positive eigenvalue of this mass matrix is

$$
\mu_{k, l}=\frac{k(l+2)}{\sqrt{2} R} .
$$

\subsection{Chain reductions}

To exemplify the quantitative differences between the quiver gauge theory defined by (3.5) and those studied in 10 which are obtained via $\mathrm{SU}(2)$-equivariant dimensional reduction over the projective line $\mathbb{C} P^{1}$, let us set $l=0$ and consider the reductions associated to the irreducible $\mathrm{SU}(3)$-representations $\underline{C}^{k, 0}$. In this case $j_{-}=0$ in (2.10), so that the monopole charges and instanton ranks are correlated as $(n, m)=(n, 3 n-2 k)$ with $n=0,1, \ldots, k$. With $p_{n}:=p_{n, 3 n-2 k}$, the explicit gauge symmetry breaking pattern is given in this limit by

$$
\mathrm{SU}(p) \longrightarrow \mathrm{U}(1)^{k} \times \prod_{n=0}^{k} \mathrm{SU}\left(p_{n}\right) \quad \text { with } \quad \sum_{n=0}^{k}(n+1) p_{n}=p
$$


Although similar to the symmetry reduction patterns of [10, the rank decompositions in (3.41) are controlled explicitly by the instanton ranks $n+1$.

From (2.41) one also finds $\Lambda_{k, 0}^{-}(n, 3 n-2 k)=0$ and $\Lambda_{k, 0}^{+}(n, 3 n-2 k)=\sqrt{(n+1)(k-n)}$. It follows that $\bar{\beta}_{n, 3 n-2 k}^{-}=0$ for all $n=0,1, \ldots, k$, and consequently all fields $\phi_{n, m}^{-}$are absent from (2.46). Thus in this case the two-dimensional quiver lattice of equivariant fields on $M$ labelled by $\mathrm{W}_{k, l}$ truncates to a one-dimensional chain



Denote $\phi_{n+1}:=\phi_{n, 3 n-2 k}^{+}$and $A^{n}:=A^{n, 3 n-2 k}$, with

$F^{n}=\mathrm{d} A^{n}+\frac{g}{\sqrt{n+1}} A^{n} \wedge A^{n} \quad$ and $\quad D \phi_{n+1}=\mathrm{d} \phi_{n+1}+g\left(\frac{1}{\sqrt{n+2}} A^{n+1} \phi_{n+1}-\frac{1}{\sqrt{n+1}} \phi_{n+1} A^{n}\right)$.

Then the action (3.5) reduces to

$$
\begin{array}{r}
S_{\mathrm{YM}}^{k, 0}=\int_{M} \mathrm{~d}^{d} x \sqrt{|G|}\left[\sum _ { n = 0 } ^ { k } \operatorname { t r } _ { p _ { n } \times p _ { n } } \left(\frac{1}{4}\left(F_{\mu \nu}^{n}\right)^{\dagger}\left(F^{n \mu \nu}\right)+\left(D_{\mu} \phi_{n+1}\right)\left(D^{\mu} \phi_{n+1}\right)^{\dagger}\right.\right. \\
\left.\left.+\left(D_{\mu} \phi_{n}\right)^{\dagger}\left(D^{\mu} \phi_{n}\right)\right)+V\left(\phi_{1}, \ldots, \phi_{k}\right)\right]
\end{array}
$$

with $\phi_{0}=\phi_{k+1}=0$ and the Higgs potential

$$
\begin{aligned}
V\left(\phi_{1}, \ldots, \phi_{k}\right)=2 g^{2} \sum_{n=0}^{k} \operatorname{tr}_{p_{n} \times p_{n}}[ & \frac{1}{n+2}\left(\frac{(n+1)(n+2)(k-n)}{4 g^{2} R^{2}} \mathbf{1}_{p_{n}}-\phi_{n+1}^{\dagger} \phi_{n+1}\right)^{2} \\
& \left.+\frac{1}{n}\left(\frac{n(n+1)(k-n+1)}{4 g^{2} R^{2}} \mathbf{1}_{p_{n}}-\phi_{n} \phi_{n}^{\dagger}\right)^{2}\right]
\end{aligned}
$$

This potential is minimized by scalar field configurations $\phi_{n}$ obeying

$$
\phi_{n}^{\dagger} \phi_{n}=\frac{n(n+1)(k-n+1)}{4 g^{2} R^{2}} \mathbf{1}_{p_{n-1}} \quad \text { and } \quad \phi_{n} \phi_{n}^{\dagger}=\frac{n(n+1)(k-n+1)}{4 g^{2} R^{2}} \mathbf{1}_{p_{n}} .
$$

In the special case where $p_{0}=p_{1}=\cdots=p_{k}=r$, so that the gauge symmetry reduction is given by

$$
\mathrm{SU}(p) \longrightarrow \mathrm{U}(1)^{k} \times \mathrm{SU}(r)^{k+1} \quad \text { with } \quad p=\frac{1}{2} r(k+1)(k+2),
$$

an explicit solution of (3.46) is given by $\phi_{n}=\phi_{n}^{0}$, where

$$
\phi_{n}^{0}=\frac{1}{2 g R} \sqrt{n(n+1)(k-n+1)} U_{n}
$$

for $n=1, \ldots, k$. The independent unitary degrees of freedom $U_{n} \in \mathrm{U}(r)$ can be removed using a $\mathrm{U}(1)^{k} \times \mathrm{SU}(r)^{k}$ gauge transformation, and this solution breaks the gauge symmetry to $\mathrm{SU}(r)$. There are $k r^{2}$ massive gauge bosons, and $k r^{2}$ physical Higgs fields represented by $r \times r$ hermitean matrices $h_{n}, n=1, \ldots, k$ with $\phi_{n}=\phi_{n}^{0}+h_{n}$. The Higgs and vector boson masses, both proportional to $\frac{1}{R}$, can be worked out by substitution into the action (3.44). A completely analogous analysis follows in the cases with $k=0$, though there will be quantitative differences. While the physics of the dynamical symmetry breaking for these systems is qualitatively analogous to the cases studied in [10, the quantitative features are significantly different due to the different forms of the 
interactions in (3.43) and of the Higgs potential in (3.45). These differences are due to the fact that while only monopole backgrounds on $\mathbb{C} P^{1}$ contribute to the equivariant dimensional reduction considered in [10], here both instanton and monopole charges on $\mathbb{C} P^{2}$ affect the quiver gauge theory.

The quantitative differences from the $\mathbb{C} P^{1}$ models are somewhat more drastic in the fermionic sector, due to the large asymmetry between the positive and negative chirality spinor harmonics on $\mathbb{C} P^{2}$ in the limit $l=0$. With the $\operatorname{spin}^{c}$ twist (3.33), the index (2.56) in this limit becomes

$$
\nu_{n}:=\nu_{n, 3 n-2 k}=\frac{1}{2}(n+1)(n-2)(2 n-1)
$$

for $n=0,1, \ldots, k$. Thus there is only a single antichiral mode $\widetilde{\psi}:=\widetilde{\psi}_{1,3-2 k ; 0}$, whose chiral partner is $\psi:=\psi_{0,-2 k ; 0}$. The remaining fermion fields $\psi_{n ; \ell}:=\psi_{n, 3 n-2 k ; \ell}$ on $M$ for $n>2$ are all induced from positive chirality spinor harmonics on $\mathbb{C} P^{2}$, transform in the fundamental representation of $\mathrm{SU}\left(p_{n}\right)$, and have gauge interactions given by

$$
\not D \psi_{n ; \ell}=\left(\not \partial_{M}-\frac{1}{2} \not \hbar+\frac{g}{\sqrt{n+1}} A^{n}\right) \psi_{n ; \ell}
$$

for each $\ell=1, \ldots, \nu_{n}$. The fermionic action (3.39) thereby truncates to

$$
S_{\mathrm{D}}^{k, 0}=\int_{M} \mathrm{~d}^{d} x \sqrt{|G|}\left[\psi^{\dagger} \not D \psi+\widetilde{\psi}^{\dagger} \not D \widetilde{\psi}+2 \sqrt{k} g\left(\psi^{\dagger} \phi_{1}^{\dagger} \gamma \widetilde{\psi}+\widetilde{\psi}^{\dagger} \phi_{1} \gamma \psi\right)+\sum_{n=3}^{k} \sum_{\ell=1}^{\nu_{n}} \psi_{n ; \ell}^{\dagger} \not D \psi_{n ; \ell}\right]
$$

and the fermion mass induced by the Higgs vacuum (3.48) and the Yukawa interaction in (3.51) is

$$
\mu_{k, 0}=\frac{\sqrt{2} k}{R} .
$$

In contrast to the Dirac-Higgs chains which arise from dimensional reduction over $\mathbb{C} P^{1}[10$, Yukawa interactions here exist for all values of $k>0$. On the other hand, there are no Yukawa interactions in (3.39) in the limit $k=0$.

Furthermore, the construction of massive eigenspinors discussed in 93.2 proceeds by substituting $\widetilde{m}=3(n-1)$ for $n=0,1, \ldots, k$ in (3.24) and involves $4(n+1)$ families of states in multi-dimensional irreducible representations of the $\mathrm{SU}(2)$ isospin group. This contrasts markedly with the situation for spinors on $\mathbb{C} P^{1}$ where all irreducible representations of the $\mathrm{U}(1)$ holonomy group are onedimensional and spinors are two-component fields, so only two families of eigenvalues ever arise from a single irreducible representation of the $\mathrm{U}(1)$ gauge group on $\mathbb{C} P^{1}$. These two families actually correspond to a single family with equally paired positive and negative eigenvalues.

\section{Dynamical symmetry breaking from the fundamental representation}

In this section we will work out the details of dynamical symmetry breaking in the quiver gauge theory which is induced by dimensional reduction from the three-dimensional fundamental representation $\underline{C}^{1,0}$ of $\mathrm{SU}(3)$. It is obtained by setting $k=1$ in the class of models studied in $\sqrt{3.3}$. The analysis in this case is completely analogous to that of the fundamental $\mathrm{SU}(2)$ representations in the $\mathbb{C} P^{1}$ models of [10]. We will determine the physical particle spectrum and masses in several explicit instances, including symmetry hierarchies which entail dynamical electroweak symmetry breaking. 


\subsection{Spontaneous symmetry breaking}

For $k=1, l=0$ there are two weights in $\mathrm{W}_{1,0}$, with $(n, m)=(1,1)$ and $(n, m)=(0,-2)$, and a single Higgs field $\phi:=\phi_{1}=\phi_{0,-2}^{+}$which is a $p_{1} \times p_{0}$ complex matrix. The quiver lattice is simply a chain consisting of one link

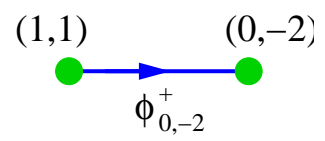

Suppose that $p_{1} \geq p_{0}$. Then, with a suitable gauge choice, the Higgs minimum can be put in the form

$$
\phi^{0}=\frac{1}{\sqrt{2} g R}\left(\begin{array}{c}
\mathbf{0}_{\left(p_{1}-p_{0}\right) \times p_{0}} \\
\mathbf{1}_{p_{0}}
\end{array}\right),
$$

where $\mathbf{0}_{\left(p_{1}-p_{0}\right) \times p_{0}}$ is a $\left(p_{1}-p_{0}\right) \times p_{0}$ matrix of zeroes. The gauge symmetry breaking sequence is given by

$\mathrm{SU}(p) \longrightarrow \mathrm{SU}\left(p_{0}\right) \times \mathrm{SU}\left(p_{1}\right) \times \mathrm{U}(1) \longrightarrow \mathrm{SU}\left(p_{1}-p_{0}\right) \times \mathrm{SU}\left(p_{0}\right)_{\operatorname{diag}} \times \mathrm{U}(1)^{\prime} \quad$ with $p=p_{0}+2 p_{1}$,

where the last step is dynamical symmetry breaking with $\mathrm{SU}\left(p_{0}\right)_{\text {diag }}$ the diagonal $\mathrm{SU}\left(p_{0}\right)$ subgroup leaving $\mathbf{1}_{p_{0}}$ invariant, and $\mathrm{U}(1)^{\prime}$ acts from the left on the top $p_{1}-p_{0}$ rows of $\phi^{0}$. The case $p_{0}>p_{1}$ can be treated similarly.

The gauge boson masses can be determined from the bicovariant derivative in (3.43), which in this case reads

$$
D \phi=\mathrm{d} \phi+g\left(\frac{1}{\sqrt{2}} A^{1} \phi-\phi A^{0}\right) .
$$

For the moment we shall take the gauge potential $A^{0}$ to lie in $\mathrm{u}\left(p_{0}\right)$ and $A^{1}$ in $\mathrm{u}\left(p_{1}\right)$, as an overall $\mathrm{u}(1)$ part will drop out. Let

$$
A^{1}=A_{L}^{a}\left(\frac{\mathrm{i} \lambda_{a}}{2}\right)+B_{L} \frac{\mathrm{i}}{\sqrt{2 p_{1}}} \mathbf{1}_{p_{1}} \quad \text { and } \quad A^{0}=A_{R}^{\tilde{a}}\left(\frac{\mathrm{i} \lambda_{\tilde{a}}}{2}\right)+B_{R} \frac{\mathrm{i}}{\sqrt{2 p_{0}}} \mathbf{1}_{p_{0}},
$$

where $\lambda_{a}$ are Gell-Mann matrices for $\mathrm{SU}\left(p_{0}\right)$ with $\operatorname{tr}_{p_{0} \times p_{0}}\left(\lambda_{a} \lambda_{b}\right)=2 \delta_{a b}, \lambda_{\tilde{a}}$ are Gell-Mann matrices for $\mathrm{SU}\left(p_{1}\right)$, and the square root factors are chosen so that the $\mathrm{U}(1)$ generators have the same normalisation as the Gell-Mann matrices. Then only the combination

$$
B:=\frac{1}{\sqrt{p}}\left(\sqrt{p_{0}} B_{L}-\sqrt{2 p_{1}} B_{R}\right)
$$

appears in (4.4), since the orthogonal combination $\frac{1}{\sqrt{p}}\left(\sqrt{2 p_{1}} B_{L}+\sqrt{p_{0}} B_{R}\right)$ decouples as it should. With this notation, the bicovariant derivative (4.4) now reads

$$
D \phi=\mathrm{d} \phi+\frac{\mathrm{i} g}{2}\left(\frac{1}{\sqrt{2}} A_{L}^{a} \lambda_{a} \phi-A_{R}^{\tilde{a}} \phi \lambda_{\tilde{a}}+\sqrt{\frac{p}{p_{1} p_{0}}} B \phi\right)
$$

from which we can obtain the gauge boson mass matrix $\boldsymbol{M}$ by substituting the vacuum expectation value (4.2) of the Higgs field to get

$$
\frac{1}{2} \boldsymbol{A}^{\top} \boldsymbol{M}^{2} \boldsymbol{A}=\operatorname{tr}_{p_{0} \times p_{0}}\left(\left(D \phi^{0}\right)^{\dagger} D \phi^{0}\right)
$$

where $\boldsymbol{A}$ is a column vector consisting of the vector bosons in (4.7). We will now work through some explicit examples. 
$p_{0}=p_{1}=r . \quad$ In this case one has

$$
\phi^{0}=\frac{1}{\sqrt{2} g R} \mathbf{1}_{r}
$$

and $\lambda_{a}=\lambda_{\tilde{a}}$. The symmetry breaking pattern is

$$
\mathrm{SU}(3 r) \longrightarrow \mathrm{SU}(r) \times \mathrm{SU}(r)_{\operatorname{diag}} \times \mathrm{U}(1)^{\prime} \longrightarrow \mathrm{SU}(r)_{\text {diag }},
$$

and only $\mathrm{SU}(r)_{\text {diag }}$ survives as a gauge symmetry. The quadratic form (4.8) is given by

$$
\operatorname{tr}_{r \times r}\left(\left(D \phi^{0}\right)^{\dagger} D \phi^{0}\right)=\frac{1}{8 R^{2}}\left[2 \delta_{a b}\left(\frac{1}{\sqrt{2}} A_{L}^{a}-A_{R}^{a}\right)\left(\frac{1}{\sqrt{2}} A_{L}^{b}-A_{R}^{b}\right)+\frac{3}{r} B^{2}\right] .
$$

The gauge boson mass matrix is thus given by

$$
\boldsymbol{M}^{2}=\frac{1}{2 R^{2}}\left(\begin{array}{ccc}
\frac{1}{2} \mathbf{1}_{r} & -\frac{1}{\sqrt{2}} \mathbf{1}_{r} & 0 \\
-\frac{1}{\sqrt{2}} \mathbf{1}_{r} & \mathbf{1}_{r} & 0 \\
0 & 0 & \frac{3}{2 r}
\end{array}\right)
$$

Diagonalising (4.12) produces massive gauge bosons $B$ together with

$$
W^{a}:=\sqrt{\frac{1}{3}} A_{L}^{a}-\sqrt{\frac{2}{3}} A_{R}^{a}
$$

with mass squared

$$
\mu_{B}^{2}=\frac{3}{4 r R^{2}} \quad \text { and } \quad \mu_{W}^{2}=\frac{3}{4 R^{2}},
$$

while the massless combinations corresponding to the unbroken symmetry group $\mathrm{SU}(r)_{\text {diag }}$ are

$$
A^{a}:=\sqrt{\frac{2}{3}} A_{L}^{a}+\sqrt{\frac{1}{3}} A_{R}^{a} .
$$

The physical Higgs fields can be incorporated into an $r \times r$ hermitean matrix $h$ with

$$
\phi=\frac{1}{\sqrt{2} g R} \mathbf{1}_{r}+h,
$$

and the Higgs boson mass read off from the term in the Higgs potential (3.45) quadratic in $h$ to get

$$
\mu_{h}^{2}=\frac{6}{R^{2}}
$$

$p_{0}=\mathbf{1}, p_{1}=\mathbf{2}$. This example exhibits $\mathrm{U}(1)$ mixing. One has $p=5$ and the pattern

$$
\mathrm{SU}(5) \longrightarrow \mathrm{SU}(2) \times \mathrm{U}(1) \longrightarrow \mathrm{U}(1)^{\prime}
$$

In this case the Higgs field $\phi$ is a two-component column vector with vacuum expectation value

$$
\phi^{0}=\frac{1}{\sqrt{2} g R}\left(\begin{array}{l}
0 \\
1
\end{array}\right) .
$$

The Higgs boson mass is again given by (4.17), but now the gauge boson mass matrix obtained from (4.7) and (4.8) mixes $A_{L}^{3}$ and $B$ as

$$
\boldsymbol{M}^{2}=\frac{1}{8 R^{2}}\left(\begin{array}{cccc}
1 & 0 & 0 & 0 \\
0 & 1 & 0 & 0 \\
0 & 0 & 1 & -\sqrt{5} \\
0 & 0 & -\sqrt{5} & 5
\end{array}\right)
$$


This gives two $W$-bosons with mass squared

$$
\mu_{W}^{2}=\frac{1}{8 R^{2}},
$$

a $Z$-boson

$$
Z=\frac{1}{\sqrt{6}}\left(A_{L}^{3}-\sqrt{5} B\right) \quad \text { with } \quad \mu_{Z}^{2}=\frac{3}{4 R^{2}},
$$

and a massless photon

$$
A=\frac{1}{\sqrt{6}}\left(\sqrt{5} A_{L}^{3}+B\right) .
$$

The Weinberg angle $\theta$ in this model is given by

$$
\sin ^{2} \theta=\frac{5}{6} .
$$

$p_{\mathbf{0}}=\mathbf{2}, \boldsymbol{p}_{\mathbf{1}}=\mathbf{1}$. Here $p=4$ and the symmetry breaking sequence (4.18) is modified to

$$
\mathrm{SU}(4) \longrightarrow \mathrm{SU}(2) \times \mathrm{U}(1) \longrightarrow \mathrm{U}(1)^{\prime} .
$$

In this case one computes

$$
\mu_{W}^{2}=\frac{1}{4 R^{2}}, \quad \mu_{Z}^{2}=\frac{3}{4 R^{2}} \quad \text { and } \quad \sin ^{2} \theta=\frac{2}{3} .
$$

This example illustrates that, in contrast to the $\mathbb{C} P^{1}$ case, the results depend on the ordering of the quiver gauge group ranks $p_{n}$.

\subsection{Fermion spectrum and Yukawa couplings}

Following the general analysis of 33.2 and 33.3 , there are two fermion zero modes $\psi_{0,-2}$ and $\widetilde{\psi}_{1,1}$ on $M$ determined by the twisting parameter $\widetilde{c}=-\frac{1}{2}$, for which the index is given by

$$
\nu_{1,1}=-1 \quad \text { and } \quad \nu_{0,-2}=+1 .
$$

The positive chirality mode on $\mathbb{C} P^{2}$ is

$$
\left.\chi_{0,-2}^{+}=|\Omega\rangle \otimes||_{0}^{0},-2\right\rangle
$$

while the negative chirality mode, which is a doublet of the $\mathrm{SU}(2)$ gauge theory on $\mathbb{C} P^{2}$, is

$$
\chi_{1,1}^{-}=\frac{1}{\sqrt{2}}\left(\sigma^{\overline{1}}|\Omega\rangle \otimes\left|{ }_{1}^{1}, 1\right\rangle+\sigma^{\overline{2}}|\Omega\rangle \otimes|\stackrel{1}{-1}, 1\rangle\right) .
$$

For example, taking $p_{0}=p_{1}=r$, we can choose the corresponding $d$-dimensional spinor fields $\widetilde{\psi}_{1,1}$ and $\psi_{0,-2}$ to transform in the fundamental representation of $\mathrm{SU}(r) \times \mathrm{SU}(r)$. After the rescalings (3.7) and (3.38), the Yukawa couplings in (3.39) for this case take the form

$$
2 g \int_{\mathbb{C} P^{2}} \beta_{\mathrm{vol}} \Psi^{\dagger}\left(\begin{array}{ccc}
0 & 0 & \phi \gamma \otimes \sigma^{\overline{1}} \\
0 & 0 & \phi \gamma \otimes \sigma^{2} \\
\phi^{\dagger} \gamma \otimes \sigma^{1} & \phi^{\dagger} \gamma \otimes \sigma^{2} & 0
\end{array}\right) \Psi=2 g\left(\widetilde{\psi}_{1,1}^{\dagger} \phi \gamma \psi_{0,-2}+\psi_{0,-2}^{\dagger} \phi^{\dagger} \gamma \widetilde{\psi}_{1,1}\right)
$$

where we have used (2.59). Expanding about the Higgs vacuum (4.9), we find a mass term for the $d$-dimensional fermions given by

$$
\frac{\sqrt{2}}{R}\left(\psi_{1,1}^{\dagger} \psi_{0,-2}+\psi_{0,-2}^{\dagger} \psi_{1,1}\right)
$$


where $\psi_{1,1}=\gamma \widetilde{\psi}_{1,1}$. This agrees with (3.52) for $k=1$.

In addition to the zero modes there is an infinite tower of massive modes. The full spectrum of the Dirac operator on $\mathbb{C} P^{2}$ can be derived using the results of $\$ 3.2$. For this, we require the irreducible $\mathrm{SU}(2) \times \mathrm{U}(1)$ representations that appear in the tensor product of the gauge group representations $(1,1)$ and $(0,-2)$ on $\mathbb{C} P^{2}$ with the spinor representation (3.22), which was shown in 3.2 to decompose as $\overline{[(1,0)]} \oplus[(0,3) \oplus \underline{(0,-3)]}$. Twisting with $c=-1$ from (3.33), to give globally well-defined bundles, alters the gauge group representations as $(1,1) \rightarrow(1,0)$ and $(0,-2) \rightarrow(0,-3)$. Thus we require the eigenvalues, and their multiplicities, of the Dirac operator for the representations

$$
\underline{(1,0)} \otimes([\underline{(1,0)}] \oplus[\underline{(0,3)} \oplus \underline{(0,-3)}])=[\underline{(2,0)} \oplus \underline{(0,0)}] \oplus[\underline{(1,3)} \oplus \underline{(1,-3)}]
$$

and

$$
\underline{(0,-3)} \otimes([\underline{(1,0)}] \oplus[\underline{(0,3)} \oplus \underline{(0,-3)}])=[\underline{(1,-3)}] \oplus[\underline{(0,0)} \oplus \underline{(0,-6)}] .
$$

The eigenvalues and their multiplicities follow from the general formulas (3.25) and (3.26) of 93.2. The eight states on the right-hand side of (4.32), a triplet, two doublets and a singlet of $\mathrm{SU}(2)$, give rise to eight infinite sequences of Dirac eigenspinors. All eigenvalues occur in equal pairs with opposite sign so there are four infinite sequences with positive eigenvalues, together with their negative eigenvalue partners. The four states on the right-hand side of (4.33), a doublet and two singlets of $\mathrm{SU}(2)$, give rise to four infinite sequences of Dirac eigenspinors with eigenvalues in equal pairs and opposite signs yielding two infinite sequences with positive eigenvalues, together with their negative eigenvalue partners. Denoting the positive eigenvalues by $\frac{\lambda_{N}}{R}$, with degeneracies $d_{N}$, the two infinite sequences arising from (4.33) are given by

$$
\begin{array}{ll}
\lambda_{N}=\sqrt{(N+1)(N+3)}, & d_{N}=(N+2)^{3}, \\
\lambda_{N}=\sqrt{(N+2)(N+3)}, & d_{N}=\frac{1}{2}(N+1)(N+4)(2 N+5)
\end{array}
$$

with $N=0,1, \ldots$ The spectrum arising from (4.32) gives two copies of (4.34), so the full spectrum consists of three copies of (4.34) together with their negative eigenvalue counterparts. The two zero modes can be thought of as coming from two copies of the first sequence in (4.34) with $N=-1$.

It can be interesting to also consider alternative values of the twisting parameter $c$, other than the choice $c=-1$ which induces Yukawa couplings in the zero mode sector of the fermionic field theory on $M$. In the present context $c=3$ gives three positive chirality zero modes, $\nu_{1,1}=3$ while $\nu_{0,-2}=0$, and $c=-3$ gives three negative chirality zero modes, $\nu_{1,1}=0$ while $\nu_{0,-2}=-3$. These zero modes could manifest themselves as three generations of fermions in the dimensionally reduced field theory.

\section{Dynamical symmetry breaking from the adjoint representation}

In this section we examine symmetry breaking from the eight-dimensional adjoint representation $\underline{C}^{1,1}$ of $\mathrm{SU}(3)$. This is the lowest representation which is qualitatively distinct from the $\mathbb{C} P^{1}$ examples, in the sense that it involves a full two-dimensional quiver lattice (2.11) of equivariant gauge fields. Again we will determine the physical particle spectrum and masses in some explicit instances. 


\subsection{Spontaneous symmetry breaking}

In the case $k=l=1$, the weight set is $\mathbf{W}_{1,1}=\{(1,3),(1,-3),(2,0),(0,0)\}$. The only non-zero coefficients $\Lambda_{1,1}^{ \pm}(n, m)$ in (2.41) are

$$
\Lambda_{1,1}^{+}(1,-3)=1, \quad \Lambda_{1,1}^{+}(0,0)=\sqrt{\frac{3}{2}}, \quad \Lambda_{1,1}^{-}(1,-3)=\sqrt{3} \quad \text { and } \quad \Lambda_{1,1}^{-}(2,0)=\sqrt{\frac{3}{2}} .
$$

Hence the only four matrix one-forms in (2.45) are

$$
\beta_{1,-3}^{ \pm}, \quad \beta_{0,0}^{+} \quad \text { and } \quad \beta_{2,0}^{-},
$$

and there are only four Higgs fields

$$
\phi_{1,-3}^{ \pm}, \quad \phi_{0,0}^{+} \quad \text { and } \quad \phi_{2,0}^{-} .
$$

The apparent asymmetry here, in that the weight $(1,3)$ does not appear while $(1,-3)$ does, is an artifact of the notation. The symmetry between the representations is clear in the quiver lattice



that indicates which $\mathrm{SU}(2) \times \mathrm{U}(1)$ representations are mapped by the Higgs field morphisms.

For illustrative purposes, we will again restrict to the case of equal quiver gauge group ranks given by $p_{1,-3}=p_{1,3}=p_{0,0}=p_{2,0}=r$ with $p=8 r$, which gives the gauge symmetry reduction pattern $\mathrm{SU}(8 r) \rightarrow \mathrm{SU}(r)^{4} \times \mathrm{U}(1)^{3}$. In this case each Higgs field $\phi_{n, m}^{ \pm}$is a square $r \times r$ matrix and the Higgs potential in (3.5), after the rescalings (3.7), is

$$
\begin{aligned}
V\left(\boldsymbol{\phi}^{+}, \boldsymbol{\phi}^{-}\right)=g^{2} \operatorname{tr}_{r \times r}[ & 3\left(\frac{3}{4 g^{2} R^{2}} \mathbf{1}_{r}-\left(\phi_{0,0}^{+}\right)^{\dagger} \phi_{0,0}^{+}\right)^{2}+\frac{5}{3}\left(\frac{3}{4 g^{2} R^{2}} \mathbf{1}_{r}-\left(\phi_{1,-3}^{+}\right)^{\dagger} \phi_{1,-3}^{+}\right)^{2} \\
& +3\left(\frac{3}{4 g^{2} R^{2}} \mathbf{1}_{r}-\left(\phi_{1,-3}^{-}\right)^{\dagger} \phi_{1,-3}^{-}\right)^{2}+\frac{5}{3}\left(\frac{3}{4 g^{2} R^{2}} \mathbf{1}_{r}-\left(\phi_{2,0}^{-}\right)^{\dagger} \phi_{2,0}^{-}\right)^{2} \\
+ & \left.\phi_{1,-3}^{+}\left(\phi_{1,-3}^{-}\right)^{\dagger}-\left.\left(\phi_{2,0}^{-}\right)^{\dagger} \phi_{0,0}^{+}\right|^{2}+\left|\phi_{0,0}^{+} \phi_{1,-3^{6}}^{-}-\phi_{2,0}^{-} \phi_{1,-3}^{+}\right|^{2}\right] . \quad(5.5)
\end{aligned}
$$

The global minimum of (5.5) is attained by setting all four Higgs fields proportional to $\mathrm{U}(r)$ matrices

$$
\phi_{n, m}^{ \pm}{ }^{0}=\frac{\sqrt{3}}{2 g R} U_{n, m}^{ \pm}
$$

which is a special instance of (3.12), together with the constraint

$$
U_{2,0}^{-} U_{1,-3}^{+}=U_{0,0}^{+} U_{1,-3}^{-} .
$$

There are therefore only three independent unitary matrices $U_{n, m}^{ \pm}$, and we can use a $\mathrm{U}(r)^{3}$ gauge transformation to set any three of them equal to $\mathbf{1}_{r}$. The constraint (5.7) then requires all four to be the identity and only the diagonal subgroup $\mathrm{SU}(r)_{\text {diag }}$ survives. The gauge symmetry is thus broken dynamically as

$$
\mathrm{SU}(8 r) \longrightarrow \mathrm{SU}(r)^{4} \times \mathrm{U}(1)^{3} \longrightarrow \mathrm{SU}(r)_{\text {diag }},
$$


with all four Higgs fields transforming in the same way under the surviving diagonal subgroup as $\phi_{n, m}^{ \pm} \rightarrow g \phi_{n, m}^{ \pm} g^{\dagger}$ where $g \in \mathrm{SU}(r)_{\text {diag }}$. Of the initial $\left(4 r^{2}-1\right)$ gauge bosons, $3 r^{2}$ become massive and of the original $8 r^{2}$ degrees of freedom in the four complex Higgs fields, $5 r^{2}$ survive as physical Higgs fields.

We can parameterise the physical Higgs fields by choosing a gauge in which three are given by hermitean matrices and one by a general complex matrix, yielding $5 r^{2}$ degrees of freedom as required. To see that such a gauge exists, we first observe that any square complex matrix has a unique polar decomposition into the product of a unitary matrix with a hermitean matrix so that, without making any gauge choice, we can always write

$$
\phi_{n, m}^{ \pm}=V_{n, m}^{ \pm}\left(\frac{\sqrt{3}}{2 g R} \mathbf{1}_{r}+h_{n, m}^{ \pm}\right)
$$

with $V_{n, m}^{ \pm}$unitary and $h_{n, m}^{ \pm}$hermitean. In this parameterisation the vacuum state (5.6) corresponds to $h_{n, m}^{ \pm}=0$ and $V_{n, m}^{ \pm}=U_{n, m}^{ \pm}$satisfying (5.7). By using an $\mathrm{SU}(r)^{4} \times \mathrm{U}(1)^{3}$ gauge transformation we can set any three of the $\mathrm{U}(r)$-valued fields $V_{n, m}^{ \pm}$to the identity, but not all four. Let us choose a gauge in which $V_{1,-3}^{ \pm}=V_{2,0}^{-}=\mathbf{1}_{r}$. In this gauge, the Higgs fields

$$
\phi_{1,-3}^{ \pm}=\frac{\sqrt{3}}{2 g R} \mathbf{1}_{r}+h_{1,-3}^{ \pm} \quad \text { and } \quad \phi_{2,0}^{-}=\frac{\sqrt{3}}{2 g R} \mathbf{1}_{r}+h_{2,0}^{-}
$$

are hermitean while

$$
\phi_{0,0}^{+}=V_{0,0}^{+}\left(\frac{\sqrt{3}}{2 g R} \mathbf{1}_{r}+h_{0,0}^{+}\right)
$$

is a general complex matrix. Although $V_{0,0}^{+}$is an arbitrary unitary field in general, the vacuum condition (5.7) in this gauge requires $U_{0,0}^{+}=\mathbf{1}_{r}$ so let us paramaterise $\phi_{0,0}^{+}$differently. Instead of (5.11), it will be more convenient to use the decomposition

$$
\phi_{0,0}^{+}=\frac{\sqrt{3}}{2 g R} \mathbf{1}_{r}+H_{0,0}^{+}+\mathrm{i} \widetilde{H}_{0,0}^{+}
$$

with $H_{0,0}^{+}$and $\widetilde{H}_{0,0}^{+}$hermitean. In this gauge the $5 r^{2}$ physical degrees of freedom in the Higgs fields are represented by the five hermitean matrices $h_{1,-3}^{ \pm}, h_{2,0}^{-}, H_{0,0}^{+}$and $\widetilde{H}_{0,0}^{+}$, and the remaining $\mathrm{SU}(r)_{\text {diag }}$ gauge degree of freedom is implemented by $\left(h_{n, m}^{ \pm}, H_{0,0}^{+}, \widetilde{H}_{0,0}^{+}\right) \rightarrow g\left(h_{n, m}^{ \pm}, H_{0,0}^{+}, \widetilde{H}_{0,0}^{+}\right) g^{\dagger}$ with $g \in \mathrm{SU}(r)_{\text {diag }}$.

The Higgs boson masses can be found by extracting the quadratic part of the potential (5.5) when expanded around the minimum. The mass matrix $\boldsymbol{M}_{h}$ works out to be given by

$$
\boldsymbol{M}_{h}^{2}=\frac{1}{2 R^{2}}\left(\begin{array}{ccccc}
3 & 0 & 0 & 0 & 0 \\
0 & 21 & -3 & 0 & 0 \\
0 & -3 & 13 & 0 & 0 \\
0 & 0 & 0 & 21 & -3 \\
0 & 0 & 0 & -3 & 13
\end{array}\right) \otimes \mathbf{1}_{r}
$$

where the rows and columns are labelled by the sequence of Higgs fields $\left\{\widetilde{H}_{0,0}^{+}, H_{0,0}^{+}, h_{1,-3}^{+}, h_{1,-3}^{-}, h_{2,0}^{-}\right\}$. There are two doubly degenerate eigenvalues

$$
\mu_{h^{ \pm}}^{2}=\frac{11}{R^{2}}
$$

corresponding to the linear combinations

$$
h^{+}=\frac{1}{\sqrt{10}}\left(h_{1,-3}^{+}-3 H_{0,0}^{+}\right) \quad \text { and } \quad h^{-}=\frac{1}{\sqrt{10}}\left(h_{2,0}^{-}-3 h_{1,-3}^{-}\right),
$$


and

$$
\mu_{h^{\prime \pm}}^{2}=\frac{6}{R^{2}}
$$

associated with the orthogonal combinations

$$
h^{\prime+}=\frac{1}{\sqrt{10}}\left(3 h_{1,-3}^{+}+H_{0,0}^{+}\right) \quad \text { and } \quad h^{\prime-}=\frac{1}{\sqrt{10}}\left(3 h_{2,0}^{-}+h_{1,-3}^{-}\right) .
$$

The lightest Higgs field is $\widetilde{H}_{0,0}^{+}$with

$$
\mu_{\widetilde{H}_{0,0}^{+}}^{2}=\frac{3}{2 R^{2}}
$$

The gauge boson masses are determined from the bicovariant derivative terms in (3.5), after the rescalings (3.7) and setting $\phi_{n, m}^{ \pm}$equal to their vacuum expectation values. Again writing the gauge potentials $A^{n, m}=\frac{\mathrm{i}}{2} A_{n, m}^{a} \lambda_{a}+\frac{\mathrm{i}}{2} A_{n, m}^{0} \sqrt{2 / r} \mathbf{1}_{r}$ in terms of Gell-Mann matrices $\lambda_{a}$ for $\mathrm{SU}(r)$ and the identity matrix, using (2.49) one finds

$$
\begin{aligned}
D \phi_{n, m}^{ \pm}=\mathrm{d} \phi_{n, m}^{ \pm}+\frac{\mathrm{i} g}{2} & \left(\frac{A_{n \pm 1, m+3}^{a}}{\sqrt{n+1 \pm 1}} \lambda_{a} \phi_{n, m}^{ \pm}-\frac{A_{n, m}^{a}}{\sqrt{n+1}} \phi_{n, m}^{ \pm} \lambda_{a}\right. \\
& \left.+\sqrt{\frac{2}{r}}\left(\frac{A_{n \pm 1, m+3}^{0}}{\sqrt{n+1 \pm 1}}-\frac{A_{n, m}^{0}}{\sqrt{n+1}}\right) \phi_{n, m}^{ \pm}\right) .
\end{aligned}
$$

By defining the normalised U(1) fields

$$
B_{n, m}^{ \pm}:=\frac{1}{\sqrt{2 n+2 \pm 1}}\left(\sqrt{n+1} A_{n \pm 1, m+3}^{0}-\sqrt{n+1 \pm 1} A_{n, m}^{0}\right)
$$

we can rewrite (5.19) as

$$
\begin{aligned}
D \phi_{n, m}^{ \pm}=\mathrm{d} \phi_{n, m}^{ \pm}+\frac{\mathrm{i} g}{2}( & \frac{A_{n \pm 1, m+3}^{a}}{\sqrt{n+1 \pm 1}} \lambda_{a} \phi_{n, m}^{ \pm}-\frac{A_{n, m}^{a}}{\sqrt{n+1}} \phi_{n, m}^{ \pm} \lambda_{a} \\
& \left.+\sqrt{\frac{2(2 n+2 \pm 1)}{r(n+1 \pm 1)(n+1)}} B_{n, m}^{ \pm} \phi_{n, m}^{ \pm}\right) .
\end{aligned}
$$

Not all four fields (5.20) are independent of course, as there are only three U(1) degrees of freedom, and indeed one has

$$
B_{1,-3}^{-}=-B_{0,0}^{+} .
$$

Now using (5.6) gives the quadratic form

$$
\begin{aligned}
\frac{1}{2} \boldsymbol{A}^{\top} \boldsymbol{M}^{2} \boldsymbol{A}=\operatorname{tr}_{r \times r}( & \left(D \phi_{1,-3}^{+}\right)^{\dagger} D \phi_{1,-3}^{+}{ }^{0}+\left(D \phi_{0,0}^{+0}\right)^{\dagger} D \phi_{0,0}^{+0} \\
& \left.+\left(D \phi_{1,-3}^{-}{ }^{0}\right)^{\dagger} D \phi_{1,-3}^{-}{ }^{0}+\left(D \phi_{2,0}^{-}{ }^{0}\right)^{\dagger} D \phi_{2,0}^{-}{ }^{0}\right)
\end{aligned}
$$

with the gauge boson mass matrix given by

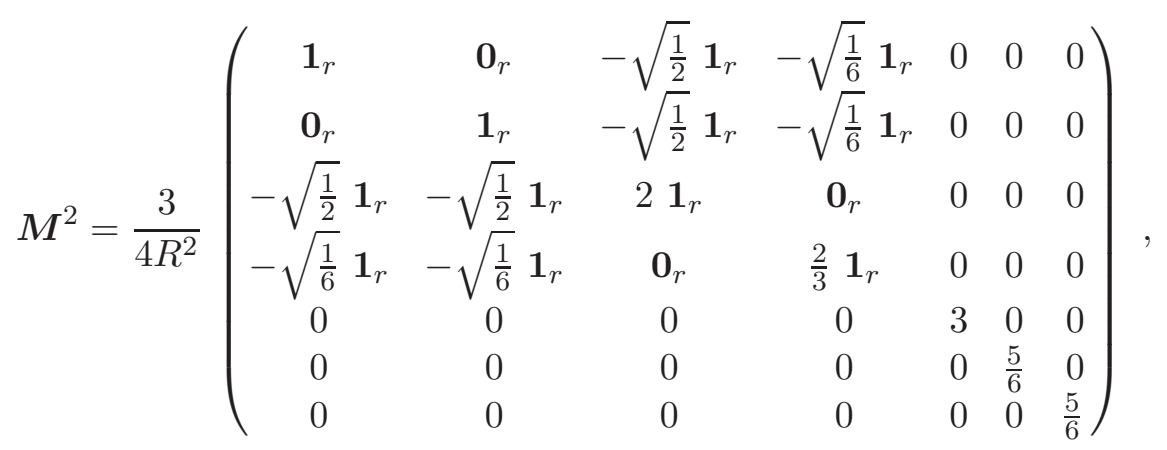


where the rows and columns of the mass matrix are ordered according to the sequence of gauge potentials $\left\{A^{1,3}, A^{1,-3}, A^{0,0}, A^{2,0}, B_{1,-3}^{-}, B_{1,-3}^{+}, B_{2,0}^{-}\right\}$. The eigenvalues of the upper left $4 \times 4$ block matrix are

$$
0, \quad \frac{2}{R^{2}} \quad \text { and } \quad \frac{3}{4 R^{2}} \quad \text { (twice) . }
$$

The linear combination

$$
A_{a}:=\frac{1}{2}\left(A_{a}^{1,3}+A_{a}^{1,-3}+\sqrt{\frac{1}{2}} A_{a}^{0,0}+\sqrt{\frac{3}{2}} A_{a}^{2,0}\right)
$$

is massless, while the gauge boson

$$
\frac{1}{2} \sqrt{\frac{1}{10}}\left(-\sqrt{6}\left(A_{a}^{1,3}+A_{a}^{1,-3}\right)+3 \sqrt{3} A_{a}^{0,0}+A_{a}^{2,0}\right)
$$

has mass squared $\frac{2}{R^{2}}$. The two linear combinations with mass squared $\frac{3}{4 R^{2}}$ are

$$
\sqrt{\frac{1}{2}}\left(A_{a}^{1,3}-A_{a}^{1,-3}\right) \quad \text { and } \quad \sqrt{\frac{1}{10}}\left(A_{a}^{1,3}+A_{a}^{1,-3}+\sqrt{2} A_{a}^{0,0}-\sqrt{6} A_{a}^{2,0}\right) .
$$

In addition, the three $\mathrm{U}(1)$ gauge bosons acquire masses given by

$$
\mu_{B_{1,-3}^{-}}^{2}=\frac{9}{4 R^{2}} \quad \text { and } \quad \mu_{B_{1,-3}^{+}}^{2}=\mu_{B_{2,0}^{-}}^{2}=\frac{5}{8 R^{2}} .
$$

It seems remarkable that the mass squared for all Higgs bosons and gauge bosons evaluate to rational multiples of $\frac{1}{R^{2}}$.

\subsection{Fermion spectrum and Yukawa couplings}

Following the analysis of $₫ 3.2$, with twisting parameter $c=-3$ there is a positive chirality zero mode associated with the $\mathrm{SU}(2)$ singlet

$$
\left.\chi_{0,0}^{+}=|\Omega\rangle \otimes||_{0}^{0}, 0\right\rangle,
$$

and a negative chirality mode associated with one of the $\mathrm{SU}(2)$ doublets

$$
\chi_{1,3}^{-}=\frac{1}{\sqrt{2}}\left(\sigma^{\overline{1}}|\Omega\rangle \otimes\left|{ }_{1}^{1}, 3\right\rangle+\sigma^{\overline{2}}|\Omega\rangle \otimes\left|{ }_{-1}^{1}, 3\right\rangle\right) .
$$

If a $(d+4)$-dimensional spinor field $\Psi$ transforms in the fundamental representation of $\mathrm{SU}(8 r)$, then the $d$-dimensional spinors $\psi_{0,0}$ and $\psi_{1,3}=\gamma \widetilde{\psi}_{1,3}$, associated with $\chi_{0,0}^{+}$and $\chi_{1,3}^{-}$respectively, transform under fundamental representations of the different $\mathrm{SU}(r)$ gauge groups with connections $A^{0,0}$ and $A^{1,3}$. When the quiver gauge symmetry is broken, they both transform under the fundamental representation of the remaining unbroken $\mathrm{SU}(r)_{\text {diag }}$ combination, with respective charges $\frac{g}{2 \sqrt{2}}$ and $\frac{g}{2}$ according to (5.26). From (3.40) it follows that the Yukawa couplings give masses $\mu_{1,1}$ to these fermions with

$$
\mu_{1,1}^{2}=\frac{9}{2 R^{2}}
$$

By (2.56), the index associated with the weight $(n, m)=(2,0)$ is zero, but the index for $(n, m)=(1,-3)$ is $\nu_{1,-3}=8$. Thus unlike the fundamental representation breaking, the adjoint representation breaking models contain massless chiral fermions. We can expect the same to be true for all representations $\underline{C}^{k, l}$ with $k+l>1$ when $l>0$, and with $k>2$ when $l=0$ (see (3.51)). 
Again there is an infinite tower of massive Dirac eigenspinors. Twisting with $c=-3$ alters the weights in $\mathrm{W}_{1,1}$ as

$(1,3) \longrightarrow(1,0), \quad(1,-3) \longrightarrow(1,-6), \quad(2,0) \longrightarrow(2,-3) \quad$ and $\quad(0,0) \longrightarrow(0,-3)$

and the corresponding $H$-modules are then tensored with the spinor representation, as in (3.24), to yield 12 irreducible holonomy group representations given by the decompositions

$$
\begin{aligned}
\underline{(1,0)} \otimes([\underline{(1,0)}] \oplus[\underline{(0,3)} \oplus \underline{(0,-3)}])=[\underline{(2,0)} \oplus \underline{(0,0)}] \oplus[\underline{(1,3)} \oplus \underline{(1,-3)}], \\
\underline{(1,-6)} \otimes([\underline{(1,0)}] \oplus[\underline{(0,3)} \oplus \underline{(0,-3)}])=[\underline{(2,-6)} \oplus \underline{(0,-6)}] \oplus[\underline{(1,-3)} \oplus \underline{(1,-9)}], \\
\underline{(2,-3)} \otimes([\underline{(1,0)}] \oplus[\underline{(0,3)} \oplus \underline{(0,-3)}])=[\underline{(3,-3)} \oplus \underline{(1,-3)}] \oplus[\underline{(2,0)} \oplus \underline{(2,-6)}], \\
\underline{(0,-3)} \otimes([\underline{(1,0)}] \oplus[\underline{(0,3)} \oplus \underline{(0,-3)}])=[\underline{(1,-3)}] \oplus \underline{(0,0)} \oplus \underline{(0,-6)}] .
\end{aligned}
$$

Since the total number of states in $\underline{C}^{1,1}$ is eight and the spinor representation (3.22) is fourdimensional, there are 32 infinite sequences corresponding to the 32 states on the right-hand side of (5.34). These consist of 16 sequences of positive eigenvalues and their negative eigenvalue partners. The 16 infinite sequences of positive eigenvalues $\frac{\lambda_{N}}{R}$, together with their degeneracies $d_{N}$, arising from the representations on the right-hand side of (5.34) can be calculated as before using (3.25) and (3.26). They are given by

$$
\begin{array}{llll}
\lambda_{N}=\sqrt{(N+1)(N+3)-2}, & d_{N}=(N+2)^{3}, & \\
\lambda_{N}=\sqrt{(N+1)(N+3)}, & d_{N}=(N+2)^{3} & (\times 3), \\
\lambda_{N}=\sqrt{(N+2)(N+3)-3}, & d_{N}=\frac{1}{2}(N+1)(N+4)(2 N+5), & \\
\lambda_{N}=\sqrt{(N+2)(N+3)-2}, & d_{N}=\frac{1}{2}(N+1)(N+4)(2 N+5) & (\times 2), \\
\lambda_{N}=\sqrt{(N+2)(N+3)}, & d_{N}=\frac{1}{2}(N+1)(N+4)(2 N+5) & (\times 3)
\end{array}
$$

and

$$
\begin{array}{lll}
\lambda_{N}=\sqrt{(N+1)(N+5)}, & d_{N}=(N+3)^{3}, \\
\lambda_{N}=\sqrt{(N+1)(N+5)+1}, & d_{N}=(N+3)^{3}, \\
\lambda_{N}=\sqrt{(N+2)(N+5)-1}, & d_{N}=\frac{1}{2}(N+2)(N+5)(2 N+7), \\
\lambda_{N}=\sqrt{(N+2)(N+5)}, & d_{N}=\frac{1}{2}(N+2)(N+5)(2 N+7), \\
\lambda_{N}=\sqrt{(N+4)^{2}-1}, & d_{N}=(N+1)(N+4)(N+7), \\
\lambda_{N}=N+4, & d_{N}=(N+1)(N+4)(N+7)
\end{array}
$$

with $N$ a non-negative integer. The two singlet zero modes are given by setting $N=-1$ in two of the three sequences in the second line of (5.35), while the octet of zero modes is gotten by taking $N=-1$ in the first sequence of (5.36).

\section{Conclusions}

We have examined in some detail the $\mathrm{SU}(3)$-equivariant dimensional reduction of pure massless Yang-Mills-Dirac theory over the coset space $\mathbb{C} P^{2}$, including a systematic incorporation of monopole and instanton backgrounds on $\mathbb{C} P^{2}$. The topologically non-trivial internal fluxes induce a Higgs 
potential as well as Yukawa couplings between the reduced fermion fields and the Higgs fields, with the standard form of dynamical symmetry breaking. For the class of models in which all Higgs fields are square matrices of the same dimension $r$, the minima of the Higgs potential have a geometrical interpretation in terms of gauge fields on the corresponding quiver lattice. As a $U(r)$ lattice gauge theory configuration, the non-abelian flux on the quiver lattice must vanish for the Higgs vacuum to be realised. Explicit examples have been presented with symmetry breaking hierarchies generated from both the fundamental and adjoint representations of $\mathrm{SU}(3)$.

For the fundamental representation models, the symmetry hierarchies

$$
\begin{aligned}
\mathrm{SU}(3 r) & \longrightarrow \mathrm{SU}(r) \times \mathrm{SU}(r) \times \mathrm{U}(1) \longrightarrow \mathrm{SU}(r) \\
\mathrm{SU}(5) & \longrightarrow \mathrm{SU}(2) \times \mathrm{U}(1) \longrightarrow \mathrm{U}(1) \\
\mathrm{SU}(4) & \longrightarrow \mathrm{SU}(2) \times \mathrm{U}(1) \longrightarrow \mathrm{U}(1)
\end{aligned}
$$

have been analysed in detail, where the first symmetry breaking is explicit, dictated by the equivariant dimensional reduction ansatz, and the second one is dynamical. Gauge boson and Higgs masses have been calculated in all three cases, and all are inversely proportional the length scale set by the metric on $\mathbb{C} P^{2}$. The complete fermion spectrum has been presented, including both chiral zero modes of the Dirac operator and massive Dirac eigenmodes. There are two zero modes, one of positive chirality $\chi_{0,-2}^{+}$and one of negative chirality $\chi_{1,1}^{-}$, which acquire masses via their Yukawa couplings (4.31), with left and right chiralities of a single massive fermion carrying different $\mathrm{SU}(2) \times \mathrm{U}(1)$ quantum numbers. This is analogous to the way that leptons and quarks acquire masses in the standard model, with the left-handed and right-handed electrons carrying different quantum numbers. The induced zero mode masses are of the same order as the mass scale of the infinite fermionic tower arising from the non-zero eigenvalues (4.34). The infinite tower may be truncated to finitely many degrees of freedom by replacing the coset space $\mathbb{C} P^{2}$ with a fuzzy projective plane $\mathbb{C} P_{F}^{2}$. However, while fuzzy versions of the line bundle zero modes $\chi_{0,-2}^{+}$ are known [17, there is as yet no explicit fuzzy construction of zero modes on instanton bundles, though one certainly exists. Models with realistic numbers of fermion generations can be obtained by changing the $\operatorname{spin}^{c}$ twisting parameter of $\$ 3.2$.

For the adjoint representation models, we examined the symmetry breaking hierarchy

$$
\mathrm{SU}(8 r) \longrightarrow \mathrm{SU}(r)^{4} \times \mathrm{U}(1)^{3} \longrightarrow \mathrm{SU}(r)
$$

in detail, calculating the gauge boson and physical Higgs masses explicitly. Again chiral zero modes $\chi_{0,0}^{+}$and $\chi_{1,3}^{-}$of the Dirac operator exist for which masses are generated by the Yukawa couplings. In this case, however, there is also an octet of positive chirality zero modes which remains exactly massless. The infinite tower of massive fermions obtained here is much more complicated than that in the case of reductions over $\mathbb{C} P^{1}$, primarily because each state of a pertinent irreducible representation of the isospin subgroup of the holonomy group of $\mathbb{C} P^{2}$ generates an infinite tower of its own. For the $\mathrm{U}(1)$ holonomy group of $\mathbb{C} P^{1}$ all irreducible representations are one-dimensional and there is only a single infinite tower for each irreducible representation, while for $\mathbb{C} P^{2}$ any given irreducible representation of $\mathrm{SU}(2)$ produces a family of infinite towers with the number of members growing as the dimension of the representation. Again these towers could be truncated by restricting to a finite number of degrees of freedom using a fuzzy regularisation on $\mathbb{C} P_{F}^{2}$.

Many of the qualititative features we have unveiled regarding the vacuum structure of field theories obtained via equivariant dimensional reduction can be expected to hold over generic homogeneous internal spaces $G / H$. The general structure of the induced quiver gauge theories is described in [7, 8]. The quiver diagram can be regarded as a lattice of dimension given by the rank of the holonomy group $H$ of the coset, and it comes with relations which equate the various 
distinct paths between any pair of vertices of the plaquettes of the quiver lattice. These relations will arise dynamically as conditions for the Higgs vacua. Thus, for instance, our lattice gauge theoretic interpretation of the Higgs minima in terms of flat connections will be a generic feature of any coset space $G / H$ for which $\operatorname{rank}(H) \geq 2$. With this in mind, it would be interesting to extend our techniques to the equivariant dimensional reductions of ten-dimensional $\mathcal{N}=1$ supersymmetric $\mathrm{E}_{8}$ gauge theories over six-dimensional coset spaces [5, 21] and of superstring theories on nearly Kähler backgrounds [22. The most interesting class of such reductions involve nonsymmetric (and nearly Kähler) six-dimensional coset spaces, with the vacua controlled by sets of torsion fluxes. Presumably these internal fluxes could be systematically incorporated, along with other topologically non-trivial background fields of the coset space, in a manner analogous to the treatment of this paper. More generally, it would be interesting to find internal coset spaces for which the equivariant dimensional reduction leads to a physical particle spectrum which is in more precise quantitative agreement with that of the standard model.

\section{Acknowledgments}

B.P.D. wishes to thank the Dublin Institute of Advanced Studies for financial support, and also the Perimeter Institute for Theoretical Physics, where this work was completed, for their hospitality and support. The work of R.J.S. was supported in part by the EU-RTN Network Grant MRTNCT-2004-005104 .

\section{A Bundles on $\mathbb{C} P^{2}$}

Consider the vector bundle $\mathcal{Q}$ over $\mathbb{C} P^{2}$ of rank two which is inverse to the line bundle $\mathcal{L}_{-1}$ with first Chern number -1 , in the K-theoretic sense

$$
\mathcal{Q} \oplus \mathcal{L}_{-1}=I^{3}
$$

where $I^{3}$ is the trivial bundle of rank three over $\mathbb{C} P^{2}$. The bundle $\mathcal{Q}$ is called a quotient bundle [20], and it has structure group $\mathrm{U}(2)$. Canonical connections on $\mathcal{L}$ and $\mathcal{Q}$ were given in (2.26) and (2.31), respectively, and indeed the construction of the flat connection $A_{0}$ in $\$ 2.3$ was based on the decomposition (A.1), see [12].

The Chern character of any bundle $\mathcal{V} \rightarrow \mathbb{C} P^{2}$ of rank $r$ can be expanded as [20]

$$
\operatorname{ch}(\mathcal{V})=r+c_{1}(\mathcal{V})+\left(\frac{1}{2} c_{1}(\mathcal{V}) \wedge c_{1}(\mathcal{V})-c_{2}(\mathcal{V})\right)
$$

where $c_{1}(\mathcal{V})$ and $c_{2}(\mathcal{V})$ are the first and second Chern characteristic classes of $\mathcal{V}$ with the integer $C_{2}(\mathcal{V})=\int_{\mathbb{C} P^{2}} c_{2}(\mathcal{V})$ the second Chern number. The rank two bundle $\mathcal{Q}$ carries $\mathrm{U}(1)$ (magnetic monopole) charge. Under the embedding $\mathrm{SU}(2) \times \mathrm{U}(1) \hookrightarrow \mathrm{SU}(3)$, the fundamental representation of SU(3) decomposes as in (2.6). This is the representation content of (A.1). The line bundle $\mathcal{L}_{-1}$ has first Chern number -1 and its fibres transform as the $H$-module $(n, m)=(0,-2)$. The $\mathrm{U}(1)$ quantum number $m$ is thus twice the Chern number of the associated line bundle and we shall call $\frac{m}{2}$ the monopole charge. The fibres of the quotient bundle $\mathcal{Q}$ transform as the $H$-module $\underline{(n, m)}=\underline{(1,1)}$. This implies that $\mathcal{Q}$ has monopole charge $\frac{1}{2}$ but first Chern number +1 , since it is of rank two and the first Chern number involves a trace, so it is equal to twice the monopole charge.

Chern characters are additive under Whitney sums of bundles, so since $\mathcal{Q} \oplus \mathcal{L}_{-1}$ is trivial we have

$$
\operatorname{ch}\left(\mathcal{Q} \oplus \mathcal{L}_{-1}\right)=\operatorname{ch}(\mathcal{Q})+\operatorname{ch}\left(\mathcal{L}_{-1}\right)=3
$$


giving $\operatorname{ch}(\mathcal{Q})=3-\operatorname{ch}\left(\mathcal{L}_{-1}\right)$. The Chern character is also multiplicative with respect to tensor products of bundles, so using (A.3) we have

$$
\operatorname{ch}\left(\mathcal{Q} \otimes \mathcal{L}_{\widetilde{b}}\right)=\operatorname{ch}(\mathcal{Q}) \wedge \operatorname{ch}\left(\mathcal{L}_{\widetilde{b}}\right)=3 \operatorname{ch}\left(\mathcal{L}_{\widetilde{b}}\right)-\operatorname{ch}\left(\mathcal{L}_{\widetilde{b}-1}\right)
$$

for any power $\widetilde{b}$. In particular, for $\widetilde{b}=-\frac{1}{2}$ we get the instanton bundle $\mathcal{I}=\mathcal{Q} \otimes \mathcal{L}_{-1 / 2}$ with

$$
\operatorname{ch}(\mathcal{I})=3 \operatorname{ch}\left(\mathcal{L}_{-1 / 2}\right)-\operatorname{ch}\left(\mathcal{L}_{-3 / 2}\right)
$$

The Chern character of the monopole line bundle $\mathcal{L}$ is $\operatorname{ch}(\mathcal{L})=\exp \xi$, where $\xi=\frac{\mathrm{i}}{2 \pi} f_{\mathrm{u}(1)}$ with $\int_{\mathbb{C} P^{2}} \xi \wedge \xi=1$, so

$$
\operatorname{ch}(\mathcal{L})=1+\xi+\frac{1}{2} \xi \wedge \xi \quad \text { and } \quad \int_{\mathbb{C} P^{2}} \operatorname{ch}(\mathcal{L})=\frac{1}{2}
$$

Similarly, one has

$$
\operatorname{ch}(\mathcal{I})=3\left(1-\frac{1}{2} \xi+\frac{1}{8} \xi \wedge \xi\right)-\left(1-\frac{3}{2} \xi+\frac{9}{8} \xi \wedge \xi\right)=2-\frac{3}{4} \xi \wedge \xi \quad \text { and } \quad \int_{\mathbb{C} P^{2}} \operatorname{ch}(\mathcal{I})=-\frac{3}{4},
$$

and hence the second Chern number of $\mathcal{I}$ is $\frac{3}{4}$, implying that $\mathcal{I}$ does not exist globally [14. Nevertheless, it plays a crucial role in the index theorem described in $\sqrt{\mathrm{C}}$.

We now have enough information to calculate the Chern characteristic classes of the rank $n+1$ instanton bundle $\mathcal{I}_{n}$. The relevant component of $\operatorname{ch}(\mathcal{I})$ for evaluating the integral over $\mathbb{C} P^{2}$ involves the square of the curvature two-form, so an explicit evaluation requires taking the trace of the second order Casimir operator in the two-dimensional vector representation of $\mathrm{SU}(2)$. The Casimir operator is $\mathrm{C}_{2}(2)=\frac{3}{4} \mathbf{1}_{2}$, and taking the trace gives a factor of 2 , so

$$
\int_{\mathbb{C} P^{2}} \operatorname{ch}(\mathcal{I})=-\frac{1}{2} C_{2}(2) \operatorname{Tr}\left(\mathbf{1}_{2}\right)
$$

The bundle

$$
\mathcal{I}_{n}:=\operatorname{Sym}^{\otimes n}(\mathcal{I})
$$

is the rank $(n+1)$ bundle given by the $n$-th symmetric tensor product of $\mathcal{I}$. As such, its second Chern number differs from (A.8) in two ways. Firstly, the dimension of the fibre is $\operatorname{Tr}\left(\mathbf{1}_{n+1}\right)$ and, secondly, the second order Casimir operator is $C_{2}(n+1)=\frac{n}{2}\left(\frac{n}{2}+1\right) \mathbf{1}_{n+1}$. From this we deduce that the second Chern number of $\mathcal{I}_{n}$ is

$$
C_{2}\left(\mathcal{I}_{n}\right)=-\int_{\mathbb{C} P^{2}} \operatorname{ch}\left(\mathcal{I}_{n}\right)=\frac{1}{2} \frac{n(n+2)}{4}(n+1)=\frac{1}{2} I(I+1)(2 I+1) .
$$

For spinor representations $\left(n=2 I\right.$ with $I \in \mathbb{Z}+\frac{1}{2}$ ) this is always fractional, while for vector representations $(n=2 I$ with $I \in \mathbb{Z})$ it is an integer corresponding to the dimension of the irreducible $\mathrm{SU}(3)$-representation $\underline{C}^{I, I-1}$.

\section{B Matrix one-form products on $\mathbb{C} P^{2}$}

We record here the explicit matrix products which are used for calculations in the quiver gauge theory of $\oint_{3}$. Using (2.45) the matrix one-form products appearing in (2.48) are given by

$$
\begin{aligned}
\bar{\beta}_{n, m}^{ \pm}{ }^{\dagger} \wedge \bar{\beta}_{n, m}^{ \pm} & =\frac{\Lambda_{k, l}^{ \pm}(n, m)^{2}}{2(n+1)} \Xi_{+}^{ \pm}(n, m ; \bar{\beta}), \\
\bar{\beta}_{n \mp 1, m-3}^{ \pm} \wedge \bar{\beta}_{n \mp 1, m-3}^{ \pm}{ }^{\dagger} & =-\frac{\Lambda_{k, l}^{ \pm}(n \mp 1, m-3)^{2}}{2(n+1 \mp 1)} \Xi_{-}^{ \pm}(n, m ; \bar{\beta}),
\end{aligned}
$$


where

$$
\begin{aligned}
\Xi_{\varepsilon}^{ \pm}(n, m ; \bar{\beta})=\sum_{q \in Q_{n}}[((n \pm & \left.q 1 \pm \varepsilon) \beta^{1} \wedge \bar{\beta}^{1}+(n \mp q+1 \pm \varepsilon) \beta^{2} \wedge \bar{\beta}^{2}\right)\left|{ }_{q}^{n}, m\right\rangle\langle\stackrel{n}{q}, m| \\
& +\sqrt{(n+1)^{2}-(q+1)^{2}} \beta^{1} \wedge \bar{\beta}^{2}\left|{ }_{q}^{n}, m\right\rangle\left\langle\begin{array}{c}
n \\
q+2
\end{array}, m\right| \\
& \left.+\sqrt{(n+1)^{2}-(q-1)^{2}} \beta^{2} \wedge \bar{\beta}^{1}\left|{ }_{q}^{n}, m\right\rangle\left\langle\begin{array}{c}
n \\
q-2
\end{array}, m\right|\right]
\end{aligned}
$$

with $\varepsilon= \pm 1$ and $\Lambda_{k, l}^{ \pm}(n, m):=0$ for $n \leq 0$. In (2.50) we encounter the matrix one-form products

$$
\left.\bar{\beta}_{n, m}^{+} \wedge \bar{\beta}_{n+1, m-3}^{-}=\left.\frac{\Lambda_{k, l}^{+}(n, m) \Lambda_{k, l}^{-}(n+1, m-3)}{\sqrt{(n+1)(n+2)}} \bar{\beta}^{1} \wedge \bar{\beta}^{2} \sum_{q \in Q_{n+1}} q\right|_{q} ^{n+1}, m+3\right\rangle\left\langle{ }_{q}^{n+1}, m-3\right|
$$

while in (2.51) we use

$$
\begin{aligned}
& \bar{\beta}_{n, m}^{+} \wedge \bar{\beta}_{n, m}^{-}{ }^{\dagger}=-\frac{\Lambda_{k, l}^{+}(n, m) \Lambda_{k, l}^{-}(n, m)}{2(n+1)} \\
& \times \sum_{q \in Q_{n+1}}\left[\left.\sqrt{(n+1)^{2}-q^{2}}\left(\beta^{1} \wedge \bar{\beta}^{1}+\beta^{2} \wedge \bar{\beta}^{2}\right)\right|_{q} ^{n+1}, m+3\right\rangle\left\langle{ }^{n-1} q, m+3\right| \\
& \left.+\left.\sqrt{(n-q)^{2}-1} \beta^{1} \wedge \bar{\beta}^{2}\right|_{q} ^{n+1}, m+3\right\rangle\left\langle\left\langle\begin{array}{l}
n-1 \\
q+2
\end{array}, m+3\right|\right. \\
& \left.\left.+\left.\sqrt{(n+q)^{2}-1} \beta^{2} \wedge \bar{\beta}^{1}\right|_{q} ^{n+1}, m+3\right\rangle\langle\stackrel{n-1}{q-2}, m+3|\right] \text {. }
\end{aligned}
$$

Using (B.1)-B.5 together with

$$
\sum_{q \in \mathrm{Q}_{n}} q=0 \quad \text { and } \quad \sum_{q \in \mathrm{Q}_{n}} q^{2}=\frac{1}{3} n(n+1)(n+2),
$$

one can derive a number of trace identities which are useful for deriving the dimensionally reduced gauge theory actions of $\$ 3$, One has

$$
\begin{aligned}
\operatorname{Tr}\left(\frac{\bar{\beta}_{n, m}^{ \pm} \Lambda^{\dagger} \wedge \star \bar{\beta}_{n, m}^{ \pm}}{\Lambda_{k, l}^{ \pm}(n, m)^{2}}\right) & =2 \pi^{2}(n+1 \pm 1) \beta_{\mathrm{vol}}, \\
\operatorname{Tr}\left(\frac{\bar{\beta}_{n, m}^{ \pm}{ }^{\dagger} \wedge \bar{\beta}_{n, m}^{ \pm} \wedge \star\left(\bar{\beta}_{n, m}^{ \pm}{ }^{\dagger} \wedge \bar{\beta}_{n, m}^{ \pm}\right)^{\dagger}}{\Lambda_{k, l}^{ \pm}(n, m)^{4}}\right) & =2 \pi^{2}(n+1 \pm 1) \beta_{\mathrm{vol}}, \\
\operatorname{Tr}\left(\frac{\bar{\beta}_{n, m}^{ \pm} \wedge \bar{\beta}_{n, m}^{ \pm} \wedge \star\left(\bar{\beta}_{n, m}^{ \pm} \wedge \bar{\beta}_{n, m}^{ \pm}\right)^{\dagger}}{\Lambda_{k, l}^{ \pm}(n, m)^{4}}\right) & =2 \pi^{2} \frac{(n+1)^{2}}{n+1 \pm 1} \beta_{\mathrm{vol}}, \\
\operatorname{Tr}\left(\frac{\bar{\beta}_{n, m}^{+} \wedge \bar{\beta}_{n+1, m-3}^{-} \wedge \star\left(\bar{\beta}_{n, m}^{+} \wedge \bar{\beta}_{n+1, m-3}^{-}\right)^{\dagger}}{\Lambda_{k, l}^{+}(n, m)^{2} \Lambda_{k, l}^{-}(n+1, m-3)^{2}}\right) & =2 \pi^{2} \frac{(n+3)}{3} \beta_{\mathrm{vol}}, \\
\operatorname{Tr}\left(\frac{\bar{\beta}_{n, m}^{+} \wedge \bar{\beta}_{n, m}^{-}{ }^{\dagger} \wedge \star\left(\bar{\beta}_{n, m}^{+} \wedge \bar{\beta}_{n, m}^{-}\right)^{\dagger}}{\Lambda_{k, l}^{+}(n, m)^{2} \Lambda_{k, l}^{-}(n, m)^{2}}\right) & =2 \pi^{2} \frac{n(n+2)}{n+1} \beta_{\mathrm{vol}},
\end{aligned}
$$

where $\operatorname{Tr}$ is the trace over $\mathrm{SU}(2)$ representations and $\star$ is the Hodge duality operator on $\mathbb{C} P^{2}$ corresponding to the metric (3.2) with

$$
\bar{\beta}^{1} \wedge \star \beta^{1}=\bar{\beta}^{2} \wedge \star \beta^{2}=\beta^{1} \wedge \star \bar{\beta}^{1}=\beta^{2} \wedge \star \bar{\beta}^{2}=2 \pi^{2} \beta_{\mathrm{vol}} .
$$

Note that $\bar{\beta}^{1} \wedge \star \bar{\beta}^{1}=\bar{\beta}^{2} \wedge \star \bar{\beta}^{2}=\beta^{1} \wedge \star \beta^{2}=\beta^{1} \wedge \star \bar{\beta}^{2}=0$, together with their hermitean conjugate equations. 


\section{Index theorem on $\mathbb{C} P^{2}$}

Spinors cannot be globally defined on $\mathbb{C} P^{2}$ due to a topological obstruction. However, globally well-defined spinors can be constructed by twisting the Dirac operator on $\mathbb{C} P^{2}$ with half-integer powers $\mathcal{L}_{\widetilde{b}}, \widetilde{b} \in \mathbb{Z}+\frac{1}{2}$ of the monopole line bundle $\mathcal{L}$. The index of the Dirac operator associated with this twisted complex is computed by the Atiyah-Singer index theorem to be [3]

$$
\nu_{b}=\int_{\mathbb{C} P^{2}} \operatorname{ch}\left(\mathcal{L}_{\widetilde{b}}\right) \wedge \widehat{A}=\frac{1}{2}(b+1)(b+2)
$$

where $\operatorname{ch}(\mathcal{L})$ is the Chern character of $\mathcal{L}, \widehat{A}$ is the Atiyah-Hirzebruch class of $\mathbb{C} P^{2}$, and $b=\widetilde{b}-\frac{3}{2}$ is an integer 4

In the main text we use the index (2.54) for higher rank $\mathrm{SU}(3)$-equivariant bundles over $\mathbb{C} P^{2}$, and we will now derive this formula here. From (A.3) the zero mode structure of the Dirac operator for spinor fields transforming under the holonomy group $H=\mathrm{SU}(2) \times \mathrm{U}(1)$, in the fundamental representation of $\mathrm{SU}(2)$ and in the background gauge field of $\mathcal{Q} \otimes \mathcal{L}_{\widetilde{b}}$, is easily evaluated [3]. Denoting this index index by $\nu_{b ; 1}$ we have, using (A.3), the formula

$$
\nu_{b ; 1}=\int_{\mathbb{C} P^{2}} \operatorname{ch}(\mathcal{Q}) \wedge \operatorname{ch}\left(\mathcal{L}_{\widetilde{b}}\right) \wedge \widehat{A}=3 \nu_{b}-\nu_{b-1}=(b+1)(b+3)
$$

where $\nu_{b}$ and $\nu_{b-1}$ have been evaluated with (C.1). The index with respect to all higher rank bundles can be computed in terms of the rank one result (C.1) by taking tensor powers of the quotient bundle $\mathcal{Q}$, since

$$
\int_{\mathbb{C} P^{2}} \operatorname{ch}\left(\mathcal{Q}^{\otimes n}\right) \wedge \operatorname{ch}\left(\mathcal{L}_{\widetilde{b}}\right) \wedge \widehat{A}=\int_{\mathbb{C} P^{2}}\left(3-\operatorname{ch}\left(\mathcal{L}_{-1}\right)\right)^{\wedge n} \wedge \operatorname{ch}\left(\mathcal{L}_{\widetilde{b}}\right) \wedge \widehat{A}
$$

There is a technical issue, however, because $\mathcal{Q}^{\otimes n}$ is a bundle of rank $2^{n}$ which is reducible in terms of $\mathrm{SU}(2)$ representations and it will be more convenient for our purposes to decompose it into irreducible representations.

The $n$-fold tensor product of the fundamental representation of $\mathrm{SU}(2) \times \mathrm{U}(1)$ decomposes into irreducible representations as

$$
\underline{(1,1)}^{\otimes n}=\bigoplus_{t=0}^{\lfloor n / 2\rfloor} N_{t, n} \underline{(n-2 t, n)},
$$

where $N_{t, n}$ is the multiplicity

$$
N_{t, n}=\frac{(n-2 t+1) n !}{(n-t+1) ! t !}
$$

Consider the equivariant rank two instanton bundle $\mathcal{I} \rightarrow \mathbb{C} P^{2}$, and its $n$-fold symmetric tensor product $\mathcal{I}_{n}$ given by (A.9) which is an equivariant vector bundle over $\mathbb{C} P^{2}$ of rank $n+1$. Its structure group is $\mathrm{SU}(2)$ and so it has no $\mathrm{U}(1)$ charge. One then has

$$
\mathcal{Q}^{\otimes n}=\left(\bigoplus_{t=0}^{\lfloor n / 2\rfloor} N_{t, n} \mathcal{I}_{n-2 t}\right) \otimes \mathcal{L}_{n / 2} .
$$

\footnotetext{
${ }^{4}$ The factor $-\frac{3}{2}$ here is essentially the power of $\mathcal{L}$ arising from the $\mathrm{U}(1)$ part of the holonomy in $\widehat{A}$. A factor of 3 is the Euler characteristic of $\mathbb{C} P^{2}$, and -3 is the first Chern number of the canonical line bundle over $\mathbb{C} P^{2}$. The factor $-\frac{3}{2}$ arises because, on a complex manifold, the spinor bundle involves the square root of the canonical line bundle. That this factor is not an integer reflects the fact that the spinor bundle over $\mathbb{C} P^{2}$ does not exist globally.
} 
In $₫ 2.5$ we use the index of the irreducible bundles $\mathcal{I}_{n} \otimes \mathcal{L}_{\widetilde{c}+m / 2}$ of $\operatorname{rank} n+1$, with $\widetilde{c} \in \mathbb{Z}+\frac{1}{2}$ a half-integer and $n \equiv m \bmod 2$ so that $\underline{(n, m)}$ is a faithful representation of $\mathrm{U}(2)$. With $\widetilde{b}=\frac{m-n}{2}+\widetilde{c}$ it is given by

$$
\nu_{b ; n}:=\int_{\mathbb{C} P^{2}} \operatorname{ch}\left(\mathcal{I}_{n}\right) \wedge \operatorname{ch}\left(\mathcal{L}_{\widetilde{b}+n / 2}\right) \wedge \widehat{A}
$$

rather than (C.3). For given $n$ this can be calculated explicitly if we know all the lower $\nu_{b ; n-2 t}$ for $t \geq 1$, since the K-theory formula

$$
\mathcal{I}_{n}=\left(\mathcal{Q}^{\otimes n} \otimes \mathcal{L}_{-n / 2}\right) \ominus\left(\bigoplus_{t=1}^{\lfloor n / 2\rfloor} N_{t, n} \mathcal{I}_{n-2 t}\right)
$$

implies

$$
\nu_{b ; n}=\int_{\mathbb{C} P^{2}} \operatorname{ch}\left(\mathcal{Q}^{\otimes n}\right) \wedge \operatorname{ch}\left(\mathcal{L}_{\widetilde{b}}\right) \wedge \widehat{A}-\sum_{t=1}^{\lfloor n / 2\rfloor} N_{t, n} \nu_{b ; n-2 t},
$$

and the first term on the right-hand side of (C.9) is known explicitly from (C.3) and (C.1). We already know $\nu_{b ; 0}=\nu_{b}$ from (C.1) and $\nu_{b ; 1}$ from (C.2), so we now have all the necessary ingredients to prove the formula (2.54) by induction on $n$.

The index $\nu_{b ; n}$ can be either positive or negative but its magnitude always corresponds to the dimension (2.4) of some irreducible representation of $\mathrm{SU}(3)$, as expected on general grounds [16. For example, if $b \geq 0$ then the index (2.54) is the dimension of the SU(3)-module $\underline{C}^{n, b}$. Under the decomposition (2.6) the irreducible $\mathrm{SU}(2) \times \mathrm{U}(1)$ representation with largest monopole charge is $\underline{(n, 2 b+n)}$, where $b+\frac{n}{2}=\widetilde{b}+\frac{n}{2}-\frac{3}{2}$ is the $\mathrm{U}(1)$ charge of the bundle $\mathcal{I}_{n} \otimes \mathcal{L}_{\widetilde{b}+n / 2}$ appearing in (C.7) including the contribution $-\frac{3}{2}$ from the Atiyah-Hirzebruch class $\widehat{A}$. We can represent this diagramatically using Young tableaux, in the notation of (2.7). The Young diagram for $\underline{C}^{n, b}$ is

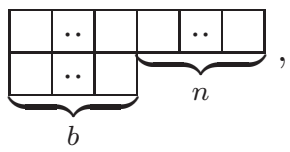

which gives the index $\nu_{b ; n}$ when $b \geq 0$. This contains the irreducible $\mathrm{SU}(2) \times \mathrm{U}(1)$ representation

$$
\begin{array}{|l|l|l|l|l|l|}
\hline \times & \cdot \cdot & \times & \times & \cdot \cdot & \times \\
\cline { 1 - 2 } \times & \cdot \cdot & \times & & \\
\cline { 1 - 2 } & &
\end{array}
$$

with $\mathrm{U}(1)$ charge $b+\frac{n}{2}$, and this is the representation content of (C.7) when $b \geq 0$.

The bundle $\mathcal{Q}^{\otimes n}$ appearing in (C.9) has monopole charge $\frac{n}{2}$, and when $n$ is odd the choice $\widetilde{b}=-\frac{n}{2}$ cancels this charge and corresponds to the pure $\mathrm{SU}(2)$ bundle $\mathcal{I}_{n}$. Hence for odd $n$ taking $b=\widetilde{b}-\frac{3}{2}=-\frac{n+3}{2}$ gives the index

$$
\nu_{-\frac{n+3}{2} ; n}=-\frac{1}{8}(n+1)^{3},
$$

and corresponds to spinors coupling to pure anti-selfdual $\mathrm{SU}(2)$ gauge fields on $\mathbb{C} P^{2}$ in the $(n+1)$ dimensional irreducible representation with no $\mathrm{U}(1)$ component. Since $n=2 k+1$ is odd this is necessarily a spinor representation of SU(2), though the magnitude of the index (C.12) corresponds to the dimension of a real representation $\underline{C}^{k, k}$ of $\mathrm{SU}(3)$. At the opposite extreme, the integer (C.1) is the index for spinors coupling to a pure U(1) self-dual gauge field on $\mathbb{C} P^{2}$ with no SU(2) component, and $\nu_{b}$ equals the dimension of the $\mathrm{SU}(3)$ representation $\underline{C}^{b, 0}$ for $b \geq 0$ while $-\nu_{b}$ equals the dimension of $\underline{C}^{0,|b|-3}$ for $b \leq-3$. 


\section{References}

[1] Th. Kaluza, Sitzungsber. Preuss. Akad. Wiss. K 1 (1921) 966; O. Klein, Z. Phys. 37 (1926) 895.

[2] T. Appelquist, A. Chodos and P.G.O. Freund, Modern Kaluza-Klein Theories (AddisonWesley, 1987); Y.A. Kubyshin, J.M. Mourao, G. Rudolph and I.P. Volobujev, Dimensional Reduction of Gauge Theories, Spontaneous Compactification and Model Building (Springer, 1989).

[3] B.P. Dolan and C. Nash, JHEP 10 (2002) 041 arXiv:hep-th/0207078]; JHEP 07 (2002) 057 arXiv:hep-th/0207007.

[4] P. Forgács and N.S. Manton, Commun. Math. Phys. 72 (1980) 15; C.H. Taubes, Commun. Math. Phys. 75 (1980) 207.

[5] D. Kapetanakis and G. Zoupanos, Phys. Rept. 219 (1992) 1.

[6] E. Witten, in: Proceedings of the 1983 Shelter Island Conference on Quantum Field Theory and the Fundamental Problems of Physics, eds. R. Jackiw, N.N. Khuri, S. Weinberg and E. Witten (MIT Press, 1985), p. 227.

[7] L. Álvarez-Cónsul and O. García-Prada, J. Reine Angew. Math. 556 (2003) 1 arXiv:math.DG/0112160; Commun. Math. Phys. 238 (2003) 1 arXiv:math.DG/0112161.

[8] O. Lechtenfeld, A.D. Popov and R.J. Szabo, Progr. Theor. Phys. Suppl. 171 (2007) 258 arXiv:0706.0979 [hep-th]].

[9] A.D. Popov and R.J. Szabo, J. Math. Phys. 47 (2006) 012306 arXiv:hep-th/0504025; O. Lechtenfeld, A.D. Popov and R.J. Szabo, JHEP 09 (2006) 054 arXiv:hep-th/0603232; A.D. Popov, Lett. Math. Phys. 84 (2008) 139 arXiv:0801.0808 [hep-th]]; Phys. Rev. D 77 (2008) 125026 arXiv:0803.3320 [hep-th]]; Mod. Phys. Lett. A 24 (2009) 349 [arXiv:0804.3845 [hep-th]].

[10] B.P. Dolan and R.J. Szabo, JHEP 03 (2009) 059 [arXiv:0901.2491 [hep-th]].

[11] P. Aschieri, J. Madore, P. Manousselis and G. Zoupanos, JHEP 04 (2004) 034 arXiv:hep-th/0310072 ; P. Aschieri, T. Grammatikopoulos, H. Steinacker and G. Zoupanos, JHEP 09 (2006) 026 [arXiv:hep-th/0606021]; D. Harland and S. Kürkçüoğlu, arXiv:0905.2338 [hep-th].

[12] O. Lechtenfeld, A.D. Popov and R.J. Szabo, JHEP 08 (2008) 093 arXiv:0806.2791 [hep-th]].

[13] J.W. Milnor and J.D. Stasheff, Characteristic Classes (Princeton University Press, 1974).

[14] B.P. Dolan, Ann. Inst. Henri Poincaré A 42 (1985) 375.

[15] C. Nash, J. Math. Phys. 27 (1986) 492.

[16] B.P. Dolan, JHEP 05 (2003) 018 arXiv:hep-th/0304037.

[17] B.P. Dolan, I. Huet, S. Murray and D. O'Connor, JHEP 03 (2008) 029 arXiv:0711.1347 [hepth]].

[18] U. Carow-Watamura, H. Steinacker and S. Watamura, J. Geom. Phys. 54 (2005) 373 arXiv:hep-th/0404130 
[19] M.B. Green, J.H. Schwarz and E. Witten, Superstring Theory: Vol. 1 (Cambridge University Press, 1987).

[20] R. Bott and L.W. Tu, Differential Forms in Algebraic Topology (Springer, 1982).

[21] P. Manousselis and G. Zoupanos, JHEP 03 (2002) 002 arXiv:hep-ph/0111125; JHEP 11 (2004) 025 arXiv:hep-ph/0406207; A. Chatzistavrakidis, P. Manousselis, N. Prezas and G. Zoupanos, Phys. Lett. B 656 (2007) 152 arXiv:0708.3222 [hep-th]]; G. Douzas, T. Grammatikopoulos and G. Zoupanos, Eur. Phys. J. C 59 (2009) 917 [arXiv:0808.3236 [hep-th]].

[22] G. Lopes Cardoso, G. Curio, G. Dall'Agata, D. Lüst, P. Manousselis and G. Zoupanos, Nucl. Phys. B 652 (2003) 5 arXiv:hep-th/0211118; P. Manousselis, N. Prezas and G. Zoupanos, Nucl. Phys. B 739 (2006) 85 [arXiv:hep-th/0511122]; A.-K. Kashani-Poor, JHEP 11 (2007) 026 arXiv:0709.4482 [hep-th]]; A. Chatzistavrakidis, P. Manousselis and G. Zoupanos, arXiv:0811.2182 [hep-th]; D. Cassani and A.-K. Kashani-Poor, Nucl. Phys. B 817 (2009) 25 arXiv:0901.4251 [hep-th]]; A. Chatzistavrakidis and G. Zoupanos, arXiv:0905.2398 [hep-th]. 